\title{
Do People Understand Monetary Policy?*
}

\author{
Carlos Carvalho \\ PUC-Rio
}

\author{
Fernanda Nechio \\ Federal Reserve Bank of San Francisco
}

July, 2012

\begin{abstract}
We combine questions from the Michigan Survey about the future path of prices, interest rates, and unemployment to investigate whether U.S. households are aware of the so-called Taylor (1993) rule. For comparison, we perform the same analysis using questions from the Survey of Professional Forecasters. Our findings support the view that some households form their expectations about the future path of interest rates, inflation, and unemployment in a way that is consistent with Taylor-type rules. The extent to which this happens, however, does not appear to be uniform across income and education levels. In particular, we find evidence that the relationship between unemployment and interest rates is not properly understood by households in the lowest income quartile, and by those with no high school diploma. We also find evidence that the perceived effect of unemployment on interest rates is asymmetric, being relevant only for interest-rate decreases. Finally, we argue that the relationships we uncover can be given a causal interpretation.
\end{abstract}

JEL classification codes: E52, E58

Keywords: survey data, monetary policy, communication, Taylor rule, inflation expectations, Michigan Survey, Survey of Professional Forecasters

\footnotetext{
*This paper was previously circulated under the title "Are People Aware of the Taylor Rule?" For comments and suggestions we thank Alessandro Barbarino, Oleg Itskhoki, Oscar Jorda, Virgiliu Midrigan, Ricardo Reis, Andrea Tambalotti, and seminar participants at NASM2012, SED2012, UC Santa Cruz, SBE 2011, EESP-FGV/SP, Brazilian Central Bank, PUC-Rio, Santa Clara University, Federal Reserve Macro System Meetings, NBER-SI 2011 (EFWW), SF Fed, EPGE/FGV Advances in Macroeconomics Workshop, and NY Fed. The views expressed in this paper are those of the authors and do not necessarily reflect the position of the Federal Reserve Bank of San Francisco or the Federal Reserve System. E-mails: cvianac@econ.puc-rio.br, fernanda.nechio@sf.frb.org.
} 


\section{Introduction}

"Improving the public's understanding of the central bank's policy strategy reduces economic and financial uncertainty and helps households and firms make more-informed decisions. Moreover, clarity about goals and strategies can help anchor the public's longer-term inflation expectations more firmly and thereby bolsters the central bank's ability to respond forcefully to adverse shocks." (Bernanke, 2010a).

Central bankers often emphasize the need to communicate with the public in order to improve its understanding of monetary policy. As the argument goes, this should allow for better-informed price- and wage-setting decisions on the part of households, and firms and improve policy effectiveness. More generally, agents' understanding of how policies that affect their decisions are conducted is perceived to be a key ingredient in the transmission mechanism of such policies. This perception is likely guided by economic theories in which the behavior of the economy depends on the interaction between the actual conduct of policy and agents' understanding of it. This interaction is well articulated in the work of Eusepi and Preston (2010), for example. In their model, if agents are not fully aware of the behavior of the central bank, policies that would otherwise guarantee stable inflation expectations might leave the door open to expectations-driven fluctuations.

In this paper we take a step back from the literature on central bank communication, expectation formation, and monetary policy effectiveness and investigate whether economic agents - households in particular - understand how monetary policy is conducted. Since the work of Taylor (1993), it became standard practice to posit that the Fed conducts monetary policy in the U.S. according to an interest-rate ("Taylor") rule that specifies a target for the policy rate as a function of deviations of inflation from its objective and some measure of slack in economic activity, such as the output gap. Estimating such policy reaction functions is the focus of a large empirical literature in monetary economics. ${ }^{1}$ Overall, Taylor-type interest-rate rules are seen as a reasonable description of how policy has been conducted in the United States during most of the time since the late 1980s. ${ }^{2}$ However, there is much less empirical work on the question of how agents perceive the conduct of monetary policy. Perhaps this is the case because these perceptions are not directly observed nor surveyed.

Our goal is to assess whether households are aware of what we refer to as the stabilization principles underlying the Taylor rule: that the policy interest rate tends to increase with inflation and to decrease with slack in economic activity. Most of the time, these principles provide a

\footnotetext{
${ }^{1}$ For a survey, see Hamalainen (2004).

${ }^{2}$ See, e.g., Judd and Rudebusch (1998). At times, however, monetary policy seems to deviate more substantially from what Taylor rules would imply (e.g., Pesenti 2008).
} 
qualitative description of how the Fed pursues its so-called dual mandate of price stability and full employment. To address this issue, we use the Survey of Consumers, conducted by Thompson Reuters and the University of Michigan ("Michigan Survey"), combining questions about the future path of prices, interest rates, and unemployment.

We adopt a simple empirical approach that, at an intuitive level, is based on the idea of separating survey answers about the paths of interest rates, inflation, and unemployment that are consistent with the stabilization principles underlying the Taylor rule from those that are not. To fix ideas, suppose that the Fed's target for the federal funds rate depends positively on contemporaneous inflation and negatively on contemporaneous unemployment and changes only with these two variables. Then, to be consistent with how the Fed conducts monetary policy, survey answers that indicate unemployment will go down and inflation will go up in one year would necessarily have to be accompanied by an answer that the Fed will tighten monetary policy in the same period. Likewise, answers that inflation will drop and unemployment will go up must be associated with a call that the Fed will ease policy.

More generally, however, an answer that is inconsistent with a particular version of the Taylor rule need not imply a misunderstanding of how monetary policy is conducted. The reason is that no specific interest-rate rule is a perfect description of policy. ${ }^{3}$ To address this issue and provide answers to our research question that can be relied on more generally, we look for consistency in households' answers in a way that is less restrictive than the test illustrated in the previous paragraph. Specifically, we test whether various empirical frequencies of households' responses about the future paths of interest rates, inflation, and unemployment differ from each other in a way that is consistent with the principles underlying the Taylor rule. To give a concrete example of our empirical approach, given a response about future inflation, we test if forecasts that interest rates will go up are more prevalent among households who predict unemployment will decline than among those who predict the opposite.

To help interpret our results for the Michigan Survey, we apply the same consistency tests using forecasts from the Survey of Professional Forecasters (SPF), conducted by the Federal Reserve Bank of Philadelphia. We take the view that professional forecasters are likely aware of how monetary policy is conducted in the United States and use results based on this survey as a reference against which to judge the findings based on the Michigan Survey.

The results we obtain are broadly consistent with the view that some U.S. households are aware

\footnotetext{
${ }^{3}$ For example, if the Taylor rule involves interest-rate smoothing (i.e., if it includes lags of the policy rate), a given forecast for inflation and unemployment can be consistent with different forecasts for the policy rate, depending on its past trajectory. The same is true if the Taylor rule specifies a reaction to variables other than inflation and unemployment or economic slack.
} 
of the principles underlying the conduct of monetary policy when forming their expectations about the future paths of interest rates, inflation, and unemployment. The degree of awareness, however, does not appear to be uniform across income and education levels, nor symmetric across predicted movements for interest rates. Our findings support the view that households are generally aware of how monetary policy tends to respond to changes in inflation. When it comes to unemployment, however, we find that answers by households in the lowest income quartile and by households with no high school diploma do not reveal the expected relationship between changes in that variable and changes in interest rates. Finally, answers by households in the upper quartile of the income distribution and households with a college degree reveal a statistically significant relationship only between increases in unemployment and decreases in interest rates.

Applying the same analysis to the SPF, we find the expected relationship between predicted changes in unemployment and predicted changes in interest rates. Surprisingly, however, we do not find statistically significant evidence of the expected relationship between predicted changes in interest rates and predicted changes in inflation. This result is troubling because in the Michigan Survey the expected relationship between interest rates and inflation shows up even in the responses of low-income and no-high-school-diploma households. We then entertain the possibility that professional forecasters consider core inflation (i.e., inflation excluding volatile components such as food and energy) to be a more important factor than headline inflation in shaping monetary policy decisions. Since 2007 the SPF includes forecasts of core inflation in addition to headline inflation. Despite the small number of observations that include forecasts of core inflation (only one year of data in our sample), our analysis based on those observations provides statistical support for the view that professional forecasters' answers are consistent with the stabilization principles underlying the Taylor rule in both of its unemployment and (core) inflation dimensions. ${ }^{4}$

While we rely on a reduced-form empirical approach, we argue that the relationships we uncover between inflation and unemployment on one side and interest rates on the other side can be given a causal interpretation. To that end, we first address the issue of endogeneity of inflation and unemployment, which arises if exogenous changes in interest rates affect these two variables. ${ }^{5}$ We argue that a realistic degree of endogeneity of inflation and unemployment should not be a problem for our analysis for two reasons. First, empirical evidence shows that exogenous changes in interest

\footnotetext{
${ }^{4}$ At its January 2012 meeting, the FOMC stated that “... inflation at the rate of 2 percent, as measured by the annual change in the price index for personal consumption expenditures, is most consistent over the longer run with the Federal Reserve's statutory mandate." However, in several earlier speeches, Fed officials highlighted core inflation as being a useful measure for inflation in the long run (e.g., Bernanke 2010 and Williams 2011). More importantly, Fed officials sometimes gave strong indications that core inflation was the relevant concept underlying its mandate to pursue price stability. For example, Bernanke (2007) states that “... the current stance of policy is likely to foster sustainable economic growth and a gradual ebbing of core inflation."

${ }^{5}$ In the monetary economics literature, these exogenous movements are often referred to as monetary or policy shocks.
} 
rates account for at most a modest fraction of the variance of inflation and unemployment at the horizons of interest (e.g., Leeper, Sims, and Zha 1996). Second, we are interested in making inferences about the signs of the causal effects of inflation and unemployment on interest rates. So, to the extent that the bias induced by endogeneity is commensurate with the fraction of the variance of inflation and unemployment that is due to exogenous changes in interest rates, it should not affect our inference. To substantiate our claim, we rely on the dynamic, stochastic, generalequilibrium model of Galí, Smets, and Wouters (2011) as a laboratory. We generate artificial data from that model, and apply our reduced-form empirical analysis to make inferences about the signs of the causal effects of inflation and unemployment on interest rates. When we use the parameters that those authors estimate using data for the U.S. economy, we find that our empirical approach recovers the correct signs of those causal effects. Corroborating our argument, we also show that our approach to inference cannot be relied upon if interest-rate shocks are excessively volatile. But in that case they explain a counterfactually large fraction of the variance of inflation and unemployment.

We then provide a simple model to illustrate how one may interpret our findings. The model allows for heterogeneity in agents' forecasts of interest rates, inflation, and unemployment, and in the way that they perceive the causal effects of the latter two variables on the former. We use the model to understand what happens when we apply our empirical approach to a pool of answers to questions about interest rates, inflation, and unemployment akin to those of the Michigan Survey in an environment with the aforementioned heterogeneity. We find that our approach recovers an average of households' perceptions of the effects of inflation and unemployment on interest rates. Finally, we discuss an important assumption underlying our interpretation, namely that households' responses to the question about interest rates are conditional on the respondents' answers about the paths for inflation and unemployment. Building on the insights of Charles Manski (e.g., Manski 2005), we illustrate and discuss how alternative assumptions might affect the interpretation that we favor.

A few recent papers investigate whether professional economists' and financial market participants' forecasts of interest rates, inflation, and output growth or some other measure of economic activity conform with Taylor-type interest-rate rules. Mitchell and Pearce (2009) analyze the Wall Street Journal's semiannual survey of professional economists, Carvalho and Minella (2009) study the Focus Survey of market participants conducted by the Brazilian Central Bank, and Fendel et al. (2011) rely on the Consensus Economic Forecast poll for the G-7 countries. These three papers estimate Taylor rules by panel regressions using numerical forecasts and address quantitative questions, such as whether the estimated coefficient on inflation is greater than unity. Schmidt and 
Nautz (2010) also use forecasts from financial market experts, but their panel data from the ZEW Financial Market Survey are categorical in nature - the expected direction of changes in interest rates, inflation, and in the economic situation in the euro zone. They focus on the accuracy of interest-rate forecasts and on decomposing forecast errors into those for inflation and economic activity and those due to misunderstanding of how the European Central Bank conducts monetary policy. These four papers are thus related to our analysis of the SPF. However, our empirical approach differs from theirs, since it is tailored to our analysis of the Michigan Survey. Finally, Hamilton et al. (2011) use the effects of macroeconomic news on fed funds futures contracts to estimate the market-perceived Taylor rule.

In Section 2 we start by describing the data we use in our analysis. Section 3 reports our results, contrasting the findings for the Michigan Survey with those for the SPF. This section also presents our analysis by demographic groups in the Michigan Survey. Section 4 addresses the issues of endogeneity and causality and presents a simple framework to interpret the results of our empirical approach. We also discuss assumptions that are important to justify the interpretations of our results. The last section concludes. The Appendix reports a series of additional results that confirm the robustness of our findings.

\section{Data and empirical approach}

\subsection{Michigan Survey}

Each month the Michigan Survey conducts telephone interviews with approximately 500 households and asks roughly 50 questions. The questionnaire covers personal finances, demographics, business conditions, and, key to this paper, it also inquires about household expectations about main economic variables, such as interest rates, inflation, and unemployment. The sample choice is statistically designed to represent all American households, and survey weights are provided to allow for inference on the population. ${ }^{6}$ Each month, an independent cross-section sample of households is selected, and some respondents are re-interviewed six months later. Under this rotating sample method, at each survey around $40 \%$ of households are being interviewed for the second time and $60 \%$ are new respondents. ${ }^{7}$ The monthly survey data begin in January 1978 . Besides the inclusion of new questions, no substantial changes have been made to the pre-existing questionnaire since that time.

\footnotetext{
${ }^{6}$ Throughout this paper, all statistics from the Michigan Survey are weighted, unless stated otherwise. Hence they refer to the U.S. population of households.

${ }^{7}$ We choose to keep the repeated interviews and assume them as independent. For robustness, we repeat all steps of the empirical analysis described below after dropping the repeated interviews, and our findings are unchanged (unreported for brevity).
} 
Our interest-rate variable corresponds to the answer to the following survey question:

"No one can say for sure, but what do you think will happen to interest rates for borrowing money during the next 12 months - will they go up, stay the same, or go down?"

For unemployment, we consider the answer to the question:

"How about people out of work during the coming 12 months - do you think that there will be more unemployment than now, about the same, or less?"

Households are not asked directly about inflation but instead about the direction of price movements and its expected size. In particular, they answer the following two questions:

"During the next 12 months, do you think that prices in general will go up, or go down, or stay where they are now?" and "By about what percent do you expect prices to go (up/down) on the average, during the next 12 months?"

We are interested in whether U.S. household perceptions of how monetary policy operates are in accordance with the principles underlying the Taylor rule. Unfortunately, the Michigan Survey does not include explicit questions about slack in economic activity - only about unemployment, which, because of fluctuations in the unemployment rate that would correspond to full employment, need not vary one-to-one with measures of economic slack. Likewise, the questions about inflation do not pertain to deviations from the Fed's (until recently unstated) inflation objective. Moreover, the questions about interest rates and unemployment refer to 12-month changes, whereas the quantitative question about the future path of prices amounts to a question about the level of 12-month inflation. Finally, the survey is not explicit about the measures that the questions pertain to.

In order to use the available questions for our study, we make the following assumptions. To deal with the fact that the question about interest rates pertains to "interest rates for borrowing money", and does not specify the measure it refers to, we simply assume that the answers to an analogous question about the policy interest rate would be the same. This is a good assumption as long as the spread between the borrowing rates that the household has in mind when answering the survey question and the policy rate does not vary too much. In our robustness analysis, presented in the Appendix, we verify that our findings are essentially unchanged if we restrict our sample to periods in which borrowing rates and the policy rate move in the same direction. Regarding the question about unemployment, we assume that the answers to an analogous question about the direction of the unemployment gap - the difference between the unemployment rate and the level of unemployment that corresponds to full employment - would be the same. This is a good assumption as long as the actual unemployment rate varies sufficiently more than the unemployment rate consistent with full employment. In our robustness analysis we use the Congressional Budget Office's estimate of the non-accelerating-inflation rate of unemployment to construct a measure of 
the unemployment gap. Our substantive findings are unchanged if we restrict our sample to periods in which unemployment and that measure of the unemployment gap move in the same direction.

The question related to the path of prices is slightly trickier. First, it does not specify a price measure. We assume it refers to the level of the headline Consumer Price Index. Second, it refers to the path for the general level of prices in the next 12 months, and not to expected changes in 12-month inflation. We thus construct artificial responses to a question about the direction of 12-month inflation by subtracting the CPI inflation in the 12 months leading up to the month of the survey from each individual response. ${ }^{8}$ Analogously with the question about unemployment, we assume that the direction of change of actual inflation maps one-to-one into the direction of change of the difference of inflation from the Fed's target. This is a sensible assumption given the Fed's mandate to pursue price stability.

Thus, conditional on our assumptions, we have answers to questions about the sign of expected 12-month changes in interest rates, inflation, and unemployment. The Michigan Survey also provides demographic characteristics about respondents. This allows us to redo our analysis conditional on specific characteristics. In particular, we will focus on levels of income, education and age.

The sample period for our analysis starts in August 1987 and ends in December 2007. The starting point coincides with the beginning of Alan Greenspan's tenure as chairman of the Federal Reserve Board, during which the Taylor rule came to be seen as a good description of U.S. monetary policy. The sample ends in December 2007 because the questions we use from the Michigan Survey pertain to 12-month forecasts, and at the end of 2008 short-term interest rates in the U.S. had essentially hit the zero bound. Moreover, as discussed above the question about interest rates in the Michigan Survey refers to borrowing rates, which diverged markedly from short-term low-risk rates during most of 2008 .

\subsection{Survey of Professional Forecasters}

The SPF is conducted at a quarterly frequency and dates from the last quarter of 1968, when it was implemented by the American Statistical Association and the National Bureau of Economic Research. Since the second quarter of 1992, the SPF has been conducted by the Federal Reserve Bank of Philadelphia. The sample size varies from year to year, with the Survey interviewing an average of 138 forecasters per year. At each quarter, respondents receive the questionnaire, which

\footnotetext{
${ }^{8}$ We convert the resulting numbers into an answer about the direction of CPI inflation by assigning a value of one when a household's 12-month inflation forecast exceeds inflation in the 12 months up to and including the month of the survey, zero if these two numbers coincide, and -1 otherwise. We convert the categorical answers about interest rates and unemployment in the same way.
} 
has to be filled and returned within a pre-established deadline. The survey covers expectations about several inflation, economic activity, unemployment, and interest-rate measures. Respondents are also required to provide their quarterly and yearly forecasts for time frames that vary from one year to three years ahead.

Unlike the Michigan Survey, the SPF asks agents about their expectations for future levels of interest rates, inflation, and unemployment. In particular, respondents are asked to provide their forecast for the next four quarters for well-specified measures of each of the three variables of interest. We focus on forecasts for the 3-month Treasury bill rate, CPI inflation, and the urban civilian unemployment rate. To make our results based on the SPF comparable to the Michigan Survey, we build categorical variables indicating whether the respondents expect the variable to move up or down or stay the same. For CPI inflation, we average across agents' forecasts for the next four quarters and subtract realized inflation in the four quarters up to and including the quarter of the survey.

\subsection{Empirical approach}

As a first step in our analysis we perform a simple test for the presence of a statistical relationship between interest rates, inflation, and unemployment. Specifically, we use the Kolmogorov-Smirnov test for equality of two distributions to test whether the empirical frequencies of responses about the direction of interest rates conditional on the responses about the direction of inflation and unemployment differ statistically from the unconditional frequencies. We then test if whatever relationship between expected movements of interest rates and expected movements of inflation and unemployment exists in the data is consistent with the stabilization principles underlying the Taylor rule.

To ease the exposition of what we actually test for, we introduce some notation. For a given pool of answers about the direction of change of interest rates, inflation, and unemployment in the subsequent 12 months, let $\mathcal{P}(x \uparrow)$ denote the fraction of answers that indicate that variable $x$ will increase. We refer to $\mathcal{P}(x)$ as the empirical frequency of predictions for 12 -month changes in $x$. Likewise, we use $\mathcal{P}(x \downarrow \mid y \uparrow, z \leftrightarrow)$ to denote the fraction of answers that indicate that $x$ will decrease in the next 12 months in the pool of answers that indicate that $y$ will increase and $z$ will remain unchanged over the same period.

With this notation, we define the partial effects of inflation and unemployment associated with 
what we refer to as the stabilization principles underlying the Taylor rule:

$$
\begin{aligned}
& \mathcal{P}(i \uparrow \mid \pi \uparrow, u)-\mathcal{P}(i \uparrow \mid \pi \leftrightarrow, u) \geq 0 ; \quad \mathcal{P}(i \uparrow \mid \pi \leftrightarrow, u)-\mathcal{P}(i \uparrow \mid \pi \downarrow, u) \geq 0, \\
& \mathcal{P}(i \downarrow \mid \pi \downarrow, u)-\mathcal{P}(i \downarrow \mid \pi \leftrightarrow, u) \geq 0 ; \quad \mathcal{P}(i \downarrow \mid \pi \leftrightarrow, u)-\mathcal{P}(i \downarrow \mid \pi \uparrow, u) \geq 0, \\
& \mathcal{P}(i \uparrow \mid \pi, u \downarrow)-\mathcal{P}(i \uparrow \mid \pi, u \leftrightarrow) \geq 0 ; \quad \mathcal{P}(i \uparrow \mid \pi, u \leftrightarrow)-\mathcal{P}(i \uparrow \mid \pi, u \uparrow) \geq 0, \\
& \mathcal{P}(i \downarrow \mid \pi, u \uparrow)-\mathcal{P}(i \downarrow \mid \pi, u \leftrightarrow) \geq 0 ; \quad \mathcal{P}(i \downarrow \mid \pi, u \leftrightarrow)-\mathcal{P}(i \downarrow \mid \pi, u \downarrow) \geq 0 .
\end{aligned}
$$

The differences in (1) and (2) are the partial effects of inflation. For example, for any given forecasted change in unemployment $u(\uparrow, \downarrow$, or $\leftrightarrow)$, going from a pool of households that predict stable inflation to a pool that predicts increasing inflation should increase the incidence of answers saying that interest rates will go up, and decrease the incidence of forecasts that interest rates will trend down. Likewise, inequalities in (3) and (4) define the partial effects of unemployment.

For each of the partial effects defined in equations (1) through (4), we set up a one-sided test where the null hypothesis is the inequality that violates the stabilization principles underlying the Taylor rule (i.e., that contradicts those partial effects). Rejection of a null hypothesis thus amounts to evidence that the particular partial effect being tested conforms with those stabilization principles. ${ }^{9}$

\section{Results}

\subsection{Realized data}

We start by illustrating our empirical approach using the actual monthly data on the 3-month Treasury bill rate, 12-month CPI inflation, and the unemployment rate for the sample period defined in Subsection 2.1. We note one caveat at the outset, that some of the conditional frequencies that we need to estimate are based on a small number of observations and some are even degenerate. Still, we choose to illustrate our empirical approach subject to these limitations, as this serves the additional purpose of providing evidence that the stabilization principles underlying the Taylor rule are discernible in the data when we apply our approach.

The top half of Table 1 documents the (unconditional) frequency of upward and downward movements in each of the three variables, whereas the bottom half of the table reports the frequency of the direction of interest-rate changes conditional on combinations of movements in inflation and unemployment. ${ }^{10}$ In line with the notation defined in Subsection 2.3, we use $\mathcal{P}(x)$ and

\footnotetext{
${ }^{9}$ An alternative would be to estimate ordered probit models. In the Appendix we briefly discuss the pros and cons of each approach and present estimates based on ordered probits.

${ }^{10}$ Episodes that involve 12-month inflation remaining constant do not appear because this is never observed in our sample.
} 
$\mathcal{P}(x \mid y \uparrow, z \downarrow, w \leftrightarrow)$ to denote, respectively, the unconditional empirical frequencies of the direction of 12-month changes in variable $x$, and the conditional empirical frequencies of the direction of 12 month changes in variable $x$ given that $y$ increases, $z$ decreases, and $w$ remains unchanged over the same period. Note that the number of observations in the conditional frequencies $\mathcal{P}(i \mid \pi \uparrow, u \leftrightarrow)$ and $\mathcal{P}(i \mid \pi \downarrow, u \leftrightarrow)$ is extremely small. In what follows, we drop these two distributions and perform our tests only for partial effects that involve changes in unemployment. Equations (5) and (6) define the partial effects of inflation and equations (7) and (8) define the partial effects unemployment after that exclusion:

$$
\begin{aligned}
& \mathcal{P}(i \uparrow \mid \pi \uparrow, u)-\mathcal{P}(i \uparrow \mid \pi \downarrow, u) \geq 0, \\
& \mathcal{P}(i \downarrow \mid \pi \downarrow, u)-\mathcal{P}(i \downarrow \mid \pi \uparrow, u) \geq 0, \\
& \mathcal{P}(i \uparrow \mid \pi, u \downarrow)-\mathcal{P}(i \uparrow \mid \pi, u \uparrow) \geq 0, \\
& \mathcal{P}(i \downarrow \mid \pi, u \uparrow)-\mathcal{P}(i \downarrow \mid \pi, u \downarrow) \geq 0 .
\end{aligned}
$$

Table 1: Empirical frequencies - realized data (\%)

\begin{tabular}{ccccc}
\hline \hline & \multicolumn{3}{c}{ Unconditional frequencies } \\
\cline { 2 - 4 } $\mathcal{P}(i)$ & $\downarrow$ & $\leftrightarrow$ & $\uparrow$ & \\
$\mathcal{P}(\pi)$ & 58.78 & - & 41.22 & \\
$\mathcal{P}(u)$ & 47.76 & - & 52.24 & \\
& 60.41 & 3.27 & 36.33 & \\
& \multicolumn{4}{c}{ Conditional frequencies } \\
\cline { 2 - 4 } $\mathcal{P}(i \mid \pi \uparrow, u \downarrow)$ & 22.78 & - & $\uparrow$ & $\#$ Obs \\
$\mathcal{P}(i \mid \pi \uparrow, u \leftrightarrow)$ & 100.00 & - & 0.00 & 79 \\
$\mathcal{P}(i \mid \pi \uparrow, u \uparrow)$ & 100.00 & - & 0.00 & 46 \\
$\mathcal{P}(i \mid \pi \downarrow, u \downarrow)$ & 43.48 & - & 56.52 & 69 \\
$\mathcal{P}(i \mid \pi \downarrow, u \leftrightarrow)$ & 80.00 & - & 20.00 & 5 \\
$\mathcal{P}(i \mid \pi \downarrow, u \uparrow)$ & 100.00 & - & 0.00 & 43 \\
\hline \hline
\end{tabular}

Table 1 presents conditional and unconditional frequencies in the data. Kolmogorov-Smirnov tests based on these frequencies suggest that, unsurprisingly, changes in interest rates, inflation, and unemployment are statistically related. ${ }^{11}$ Table 2 reports one-sided tests of the partial effects of inflation and unemployment. ${ }^{12}$ Note that we use overlapping 12-month periods, and thus we compute standard errors and test statistics that account for serial correlation using the Newey-West approach. In the absence of an obvious guide to pick the correlation lag length, we obtain standard errors with correction for autocorrelation ranging from 1 to 12 months. For consistency, throughout

\footnotetext{
${ }^{11}$ For brevity we do not report these results, which are available upon request.

${ }^{12}$ The entries with dashes correspond to cases that involve degenerate distributions. The symmetry in the table comes from the fact that, in the data, interest rates always move (either up or down) in 12-month periods, and so the events $i \uparrow \mid \cdot$ and $i \downarrow \mid \cdot$ constitute a partition of the universe of possible outcomes in all of the conditional distributions.
} 
the paper we report standard errors that account for residuals correlation of up to 12 lags. ${ }^{13}$ All of the partial effects are statistically significant and in line with the principles underlying the Taylor rule.

Table 2: Partial effects - realized data

\begin{tabular}{|c|c|c|c|}
\hline \multicolumn{4}{|c|}{ Partial effects of inflation } \\
\hline Null Hypothesis & mean diff & t-stat & p-value \\
\hline $\mathcal{P}(i \uparrow \mid \pi \downarrow, u \downarrow) \geq \mathcal{P}(i \uparrow \mid \pi \uparrow, u \downarrow)$ & 0.21 & 2.41 & 0.01 \\
\hline $\mathcal{P}(i \uparrow \mid \pi \downarrow, u \uparrow) \geq \mathcal{P}(i \uparrow \mid \pi \uparrow, u \uparrow)$ & - & - & - \\
\hline $\mathcal{P}(i \downarrow \mid \pi \uparrow, u \downarrow) \geq \mathcal{P}(i \downarrow \mid \pi \downarrow, u \downarrow)$ & 0.21 & 2.41 & 0.01 \\
\hline $\mathcal{P}(i \downarrow \mid \pi \uparrow, u \uparrow) \geq \mathcal{P}(i \downarrow \mid \pi \downarrow, u \uparrow)$ & - & - & - \\
\hline \multicolumn{4}{|c|}{ Partial effects of unemployment } \\
\hline Null Hypothesis & mean diff & t-stat & p-value \\
\hline $\mathcal{P}(i \uparrow \mid \pi \downarrow, u \uparrow) \geq \mathcal{P}(i \uparrow \mid \pi \downarrow, u \downarrow)$ & 0.57 & 3.24 & 0.00 \\
\hline $\mathcal{P}(i \uparrow \mid \pi \uparrow, u \uparrow) \geq \mathcal{P}(i \uparrow \mid \pi \uparrow, u \downarrow)$ & 0.77 & 5.37 & 0.00 \\
\hline $\mathcal{P}(i \downarrow \mid \pi \downarrow, u \downarrow) \geq \mathcal{P}(i \downarrow \mid \pi \downarrow, u \uparrow)$ & 0.57 & 3.24 & 0.00 \\
\hline $\mathcal{P}(i \downarrow \mid \pi \uparrow, u \downarrow) \geq \mathcal{P}(i \downarrow \mid \pi \uparrow, u \uparrow)$ & 0.77 & 5.37 & 0.00 \\
\hline
\end{tabular}

\subsection{Michigan Survey}

Table 3 reports the unconditional and conditional frequencies of households' responses regarding the expected changes in interest rates, inflation, and unemployment, in analogy to Table 1. A striking difference relative to the empirical distributions based on the realized data is the unconditional frequency of responses about the direction of changes in unemployment. While households got it roughly right that unemployment would increase slightly more than one-third of the time during our sample, more than half of their responses indicated that unemployment would be unchanged after 12 months - whereas in reality this only happened around $3 \%$ of the time. The discrepancy in the distribution of answers about changes in interest rates is smaller but also noteworthy. Answers about inflation fare better in this comparison. ${ }^{14}$

The bottom half of Table 3 reports the conditional frequencies of movements in interest rates based on households' responses. They also appear to be quite different from the corresponding empirical distributions based on realized data. These differences may reflect poor forecasting per-

\footnotetext{
${ }^{13}$ Statistical tests with Newey-West standard errors using lags that vary from 1 to 12 months yield essentially the same results, and are available upon request.

${ }^{14}$ These discrepancies notwithstanding, the Michigan Survey provides evidence that answers to those two questions provide useful information about economic developments over time. In particular, the difference between the fraction of responses predicting increases and the fraction of responses predicting decreases in interest rates and unemployment commove quite strongly with, respectively, the annual percentage point change in the prime borrowing rate and the annual percentage point change in the unemployment rate. See the material about the Survey available in http://www.sca.isr.umich.edu/documents.php?c=i (file Survey Description). For a critical view of the informational content of the answers regarding unemployment, see Tortorice (2011).
} 
formance and/or poor understanding of the relationship between inflation and unemployment on one side and interest rates on the other side. In what follows we focus on whether we can use these answers to tease out information about how households perceive that relationship using the partial effects defined in the previous section. This question is of interest irrespective of whether households are good forecasters.

Table 3: Frequencies of responses - Michigan Survey (\%)

\begin{tabular}{ccccc}
\hline \hline & \multicolumn{3}{c}{ Unconditional frequencies } \\
\cline { 2 - 4 } $\mathcal{P}(i)$ & $\downarrow$ & $\leftrightarrow$ & $\uparrow$ \\
$\mathcal{P}(\pi)$ & 14.54 & 28.46 & 57.00 & \\
$\mathcal{P}(u)$ & 14.47 & - & 53.53 & \\
& \multicolumn{4}{c}{ Conditional frequencies } \\
\cline { 2 - 4 } & $\downarrow$ & $\leftrightarrow$ & $\uparrow$ & \#Obs* \\
$\mathcal{P}(i \mid \pi \uparrow, u \downarrow)$ & 12.73 & 26.36 & 60.90 & 7,411 \\
$\mathcal{P}(i \mid \pi \uparrow, u \leftrightarrow)$ & 10.72 & 28.73 & 60.55 & 27,611 \\
$\mathcal{P}(i \mid \pi \uparrow, u \uparrow)$ & 15.19 & 22.36 & 62.45 & 21,651 \\
$\mathcal{P}(i \mid \pi \downarrow, u \downarrow)$ & 15.32 & 33.05 & 51.63 & 7,736 \\
$\mathcal{P}(i \mid \pi \downarrow, u \leftrightarrow)$ & 14.48 & 33.61 & 51.90 & 27,084 \\
$\mathcal{P}(i \mid \pi \downarrow, u \uparrow)$ & 21.34 & 26.28 & 52.39 & 14,884 \\
\hline \hline *Unweighted number of observations. &
\end{tabular}

Table 4 reports one-sided tests of the partial effects of inflation and unemployment perceived by households, given in equations (1) through (4). All of the partial effects of inflation are statistically significant, and in line with the principles underlying the Taylor rule. The same is not true of the partial effects of unemployment. In fact, only two out of the eight partial effects that we test for are consistent with the principles underlying the Taylor rule, and some of the tests actually provide statistically significant evidence in favor of partial effects with the opposite sign.

However, results for the partial effects of unemployment might be driven by distributions of responses that involve predictions of stable unemployment $(u \leftrightarrow)$. Indeed, all of the partial effects rely on such distributions. To check whether they drive the results for the partial effects of unemployment, we redo the tests based on partial effects that only involve distributions derived from answers that unemployment will move up or down, as described by equations (5) through (8).

The results of the one-sided tests are presented in Table 5 (for completeness we also redo the tests for the partial effects of inflation). They show that the statistically significant evidence against the perceived partial effects of unemployment being consistent with the Taylor rule does not hinge on the pools of answers that involve forecasts of stable unemployment.

We conclude that households' perceived partial effects of inflation are consistent with the principles underlying the Taylor rule in all cases. For unemployment, however, this is not the case. There is statistical evidence that some of the partial effects of unemployment are consistent with those principles - those that pertain to interest-rate decreases. But there is also evidence that some of the perceived partial effects of unemployment are opposite to what the principles underlying the 
Table 4: Partial effects - Michigan Survey

\begin{tabular}{lccc}
\hline \hline \multicolumn{4}{c}{ Partial effects of inflation } \\
\hline Null Hypothesis: & mean diff. & test stat. & p-value \\
$\mathcal{P}(i \uparrow \mid \pi \downarrow, u \downarrow) \geq \mathcal{P}(i \uparrow \mid \pi \uparrow, u \downarrow)$ & 0.10 & 10.21 & 0.00 \\
$\mathcal{P}(i \uparrow \mid \pi \downarrow, u \leftrightarrow) \geq \mathcal{P}(i \uparrow \mid \pi \uparrow, u \leftrightarrow)$ & 0.09 & 17.59 & 0.00 \\
$\mathcal{P}(i \uparrow \mid \pi \downarrow, u \uparrow) \geq \mathcal{P}(i \uparrow \mid \pi \uparrow, u \uparrow)$ & 0.10 & 16.09 & 0.00 \\
& & & \\
$\mathcal{P}(i \downarrow \mid \pi \uparrow, u \downarrow) \geq \mathcal{P}(i \downarrow \mid \pi \downarrow, u \downarrow)$ & 0.03 & 4.10 & 0.00 \\
$\mathcal{P}(i \downarrow \mid \pi \downarrow, u \leftrightarrow) \geq \mathcal{P}(i \downarrow \mid \pi \uparrow, u \leftrightarrow)$ & 0.04 & 11.46 & 0.00 \\
$\mathcal{P}(i \downarrow \mid \pi \uparrow, u \uparrow) \geq \mathcal{P}(i \downarrow \mid \pi \downarrow, u \uparrow)$ & 0.06 & 12.70 & 0.00 \\
& & & \\
$\quad \quad P a r t i a l$ effects of unemployment & & \\
& mean diff. & test stat. & p-value \\
Null Hypothesis: & 0.00 & -0.80 & 0.79 \\
$\mathcal{P}(i \uparrow \mid \pi \downarrow, u \uparrow) \geq \mathcal{P}(i \uparrow \mid \pi \downarrow, u \leftrightarrow)$ & -0.02 & -3.66 & 1.00 \\
$\mathcal{P}(i \uparrow \mid \pi \uparrow, u \uparrow) \geq \mathcal{P}(i \uparrow \mid \pi \uparrow, u \leftrightarrow)$ & -0.00 & 0.65 \\
$\mathcal{P}(i \uparrow \mid \pi \downarrow, u \leftrightarrow) \geq \mathcal{P}(i \uparrow \mid \pi \downarrow, u \downarrow)$ & -0.01 & -0.38 & 0.31 \\
$\mathcal{P}(i \uparrow \mid \pi \uparrow, u \leftrightarrow) \geq \mathcal{P}(i \uparrow \mid \pi \uparrow, u \uparrow)$ & 0.00 & 0.48 & \\
& & & \\
$\mathcal{P}(i \downarrow \mid \pi \downarrow, u \downarrow) \geq \mathcal{P}(i \downarrow \mid \pi \downarrow, u \leftrightarrow)$ & -0.01 & -1.61 & 0.95 \\
$\mathcal{P}(i \downarrow \mid \pi \uparrow, u \downarrow) \geq \mathcal{P}(i \downarrow \mid \pi \uparrow, u \leftrightarrow)$ & -0.02 & -4.15 & 1.00 \\
$\mathcal{P}(i \downarrow \mid \pi \downarrow, u \leftrightarrow) \geq \mathcal{P}(i \downarrow \mid \pi \downarrow, u \uparrow)$ & 0.07 & 14.79 & 0.00 \\
$\mathcal{P}(i \downarrow \mid \pi \uparrow, u \leftrightarrow) \geq \mathcal{P}(i \downarrow \mid \pi \uparrow, u \uparrow)$ & 0.05 & 12.51 & 0.00 \\
\hline \hline
\end{tabular}

Test statistics and p-values are based on Newey-West standard errors with correction for up to 12 lags.

Table 5: Partial effects after "dropping $i \mid \pi, u \leftrightarrow$ "- Michigan Survey

\begin{tabular}{lccc}
\hline \hline \multicolumn{4}{c}{ Partial effects of inflation } \\
\hline Null Hypothesis & mean diff & t-stat & p-value \\
$\mathcal{P}(i \uparrow \mid \pi \downarrow, u \downarrow) \geq \mathcal{P}(i \uparrow \mid \pi \uparrow, u \downarrow)$ & 0.10 & 10.23 & 0.00 \\
$\mathcal{P}(i \uparrow \mid \pi \downarrow, u \uparrow) \geq \mathcal{P}(i \uparrow \mid \pi \uparrow, u \uparrow)$ & 0.10 & 16.09 & 0.00 \\
$\mathcal{P}(i \downarrow \mid \pi \uparrow, u \downarrow) \geq \mathcal{P}(i \downarrow \mid \pi \downarrow, u \downarrow)$ & 0.03 & 4.09 & 0.00 \\
$\mathcal{P}(i \downarrow \mid \pi \uparrow, u \uparrow) \geq \mathcal{P}(i \downarrow \mid \pi \downarrow, u \uparrow)$ & 0.06 & 12.75 & 0.00 \\
\multicolumn{5}{c}{ Partial effects of unemployment } \\
\hline Null Hypothesis $\quad$ mean diff & t-stat & p-value \\
$\mathcal{P}(i \uparrow \mid \pi \downarrow, u \uparrow) \geq \mathcal{P}(i \uparrow \mid \pi \downarrow, u \downarrow)$ & -0.01 & -0.94 & 0.83 \\
$\mathcal{P}(i \uparrow \mid \pi \uparrow, u \uparrow) \geq \mathcal{P}(i \uparrow \mid \pi \uparrow, u \downarrow)$ & -0.01 & -2.06 & 0.98 \\
$\mathcal{P}(i \downarrow \mid \pi \downarrow, u \downarrow) \geq \mathcal{P}(i \downarrow \mid \pi \downarrow, u \uparrow)$ & 0.05 & 9.98 & 0.00 \\
$\mathcal{P}(i \downarrow \mid \pi \uparrow, u \downarrow) \geq \mathcal{P}(i \downarrow \mid \pi \uparrow, u \uparrow)$ & 0.02 & 4.74 & 0.00 \\
\hline \hline
\end{tabular}

Test statistics and p-values are based on Newey-West standard errors with correction for up to 12 lags. 
Taylor rule imply. These results might suggest that households do not perceive the relationship between unemployment and interest rates symmetrically, failing to realize the effects that tightening labor market conditions appear to have on the likelihood of interest-rate increases. We explore this possibility further in the next subsection, where we redo our analysis pooling answers according to households' demographic characteristics.

\subsubsection{Demographics}

We first focus on income and education levels, and then provide results by age groups. We start by comparing results for the lowest and highest income quartiles, and for the groups of respondents with no high school diploma, and those who have at least a college degree. For brevity we present the conditional and unconditional frequencies of responses in the Appendix, and exclude results that involve forecasts of stable unemployment (results are robust to using those answers as well).

The first part of Table 6 shows the partial effects of inflation by, respectively, income and education levels. With few exceptions, they are statistically significant at the usual levels and in accordance with the principles underlying the Taylor rule. The same is not true of the partial effects of unemployment. The second part of Table 6 shows that for low-income households there is only one statistically significant partial effect with the expected sign. In fact, two of the partial effects of unemployment for this demographic group show up as statistically significant with a sign that contradicts the stabilization principles underlying the Taylor rule. In contrast, all but one of the partial effects of unemployment come out as expected for households in the highest income quartile. Turning to education, for households without a high school diploma only one partial effect of unemployment is statistically significant with the expected sign. In contrast, both partial effects of unemployment for interest-rate decreases come out as expected for households with at least a college degree.

Among the several additional demographic characteristics that the Michigan Survey provides, we report in Table 7 the partial effects of inflation and unemployment across age intervals of the head of the household. Heads with a higher age profile have experienced different economic environments and are more likely to have learned about monetary policy over time. Indeed, the table shows that

the highest age bracket is the only one for which all of the partial effects of unemployment come out consistent with the stabilization principles underlying the Taylor rule.

\subsection{Survey of professional forecasters}

We now turn to the SPF. The top half of Table 8 reports the unconditional frequencies of professional forecasters' responses regarding the direction of 12-month changes in interest rates, inflation, and unemployment, while the bottom half of the table reports the conditional frequencies of answers regarding interest-rate changes. In line with the realized data, note the small number of observations for distributions that involve forecasts of stable unemployment. In what follows we exclude results that involve these forecasts.

Table 9 reports one-sided tests of the partial effects of inflation and unemployment perceived 
Table 6: Partial effects by income and education

\begin{tabular}{|c|c|c|c|c|c|c|}
\hline \multicolumn{7}{|c|}{ Partial effects of inflation } \\
\hline & \multicolumn{3}{|c|}{ Lowest income quartile } & \multicolumn{3}{|c|}{ No high school diploma } \\
\hline Null Hypothesis & mean diff. & test stat. & p-value & mean diff. & test stat. & p-value \\
\hline $\mathcal{P}(i \uparrow \mid \pi \downarrow, u \downarrow) \geq \mathcal{P}(i \uparrow \mid \pi \uparrow, u \downarrow)$ & 0.12 & 5.63 & 0.00 & 0.11 & 3.51 & 0.00 \\
\hline $\mathcal{P}(i \uparrow \mid \pi \downarrow, u \uparrow) \geq \mathcal{P}(i \uparrow \mid \pi \uparrow, u \uparrow)$ & 0.10 & 7.58 & 0.00 & 0.11 & 5.68 & 0.00 \\
\hline $\mathcal{P}(i \downarrow \mid \pi \uparrow, u \downarrow) \geq \mathcal{P}(i \downarrow \mid \pi \downarrow, u \downarrow)$ & 0.04 & 1.98 & 0.02 & 0.03 & 1.11 & 0.13 \\
\hline \multirow[t]{2}{*}{$\mathcal{P}(i \downarrow \mid \pi \uparrow, u \uparrow) \geq \mathcal{P}(i \downarrow \mid \pi \downarrow, u \uparrow)$} & 0.06 & 6.02 & 0.00 & 0.06 & 3.70 & 0.00 \\
\hline & \multicolumn{3}{|c|}{ Highest income quartile } & \multicolumn{3}{|c|}{ At least college degree } \\
\hline Null Hypothesis & Mean diff & t-stat & p-value & Mean diff & t-stat & $\mathrm{p}$-value \\
\hline $\mathcal{P}(i \uparrow \mid \pi \downarrow, u \downarrow) \geq \mathcal{P}(i \uparrow \mid \pi \uparrow, u \downarrow)$ & 0.10 & 5.36 & 0.00 & 0.09 & 5.65 & 0.00 \\
\hline $\mathcal{P}(i \uparrow \mid \pi \downarrow, u \uparrow) \geq \mathcal{P}(i \uparrow \mid \pi \uparrow, u \uparrow)$ & 0.12 & 10.07 & 0.00 & 0.10 & 10.29 & 0.00 \\
\hline $\mathcal{P}(i \downarrow \mid \pi \uparrow, u \downarrow) \geq \mathcal{P}(i \downarrow \mid \pi \downarrow, u \downarrow)$ & 0.02 & 2.02 & 0.02 & 0.03 & 2.68 & 0.00 \\
\hline $\mathcal{P}(i \downarrow \mid \pi \uparrow, u \uparrow) \geq \mathcal{P}(i \downarrow \mid \pi \downarrow, u \uparrow)$ & 0.07 & 7.68 & 0.00 & 0.07 & 9.40 & 0.00 \\
\hline \multicolumn{7}{|c|}{ Partial effects of unemployment } \\
\hline & \multicolumn{3}{|c|}{ Lowest income quartile } & \multicolumn{3}{|c|}{ No high school diploma } \\
\hline Null Hypothesis & mean diff. & test stat. & $\mathrm{p}$-value & mean diff. & test stat. & p-value \\
\hline $\mathcal{P}(i \uparrow \mid \pi \downarrow, u \uparrow) \geq \mathcal{P}(i \uparrow \mid \pi \downarrow, u \downarrow)$ & -0.07 & -3.36 & 1.00 & -0.03 & -0.93 & 0.82 \\
\hline $\mathcal{P}(i \uparrow \mid \pi \uparrow, u \uparrow) \geq \mathcal{P}(i \uparrow \mid \pi \uparrow, u \downarrow)$ & -0.05 & -2.72 & 1.00 & -0.03 & -1.08 & 0.86 \\
\hline $\mathcal{P}(i \downarrow \mid \pi \downarrow, u \downarrow) \geq \mathcal{P}(i \downarrow \mid \pi \downarrow, u \uparrow)$ & 0.03 & 2.60 & 0.00 & 0.02 & 1.83 & 0.03 \\
\hline \multirow[t]{2}{*}{$\mathcal{P}(i \downarrow \mid \pi \uparrow, u \downarrow) \geq \mathcal{P}(i \downarrow \mid \pi \uparrow, u \uparrow)$} & 0.00 & 0.42 & 0.34 & 0.00 & 0.42 & 0.34 \\
\hline & \multicolumn{3}{|c|}{ Highest income quartile } & \multicolumn{3}{|c|}{ At least college degree } \\
\hline Null Hypothesis & Mean diff & t-stat & $\mathrm{p}$-value & Mean diff & t-stat & p-value \\
\hline $\mathcal{P}(i \uparrow \mid \pi \downarrow, u \uparrow) \geq \mathcal{P}(i \uparrow \mid \pi \downarrow, u \downarrow)$ & 0.03 & 3.74 & 0.00 & 0.03 & 2.56 & 0.01 \\
\hline $\mathcal{P}(i \uparrow \mid \pi \uparrow, u \uparrow) \geq \mathcal{P}(i \uparrow \mid \pi \uparrow, u \downarrow)$ & 0.01 & 1.80 & 0.04 & 0.01 & 0.96 & 0.17 \\
\hline $\mathcal{P}(i \downarrow \mid \pi \downarrow, u \downarrow) \geq \mathcal{P}(i \downarrow \mid \pi \downarrow, u \uparrow)$ & 0.09 & 9.78 & 0.00 & 0.09 & 10.50 & 0.00 \\
\hline $\mathcal{P}(i \downarrow \mid \pi \uparrow, u \downarrow) \geq \mathcal{P}(i \downarrow \mid \pi \uparrow, u \uparrow)$ & 0.04 & 5.48 & 0.00 & 0.05 & 5.97 & 0.00 \\
\hline
\end{tabular}

Test statistics and p-values are based on Newey-West standard errors with correction for up to 12 lags. 
Table 7: Partial effects by age intervals

\begin{tabular}{|c|c|c|c|c|c|c|}
\hline \multicolumn{7}{|c|}{ Partial effects of inflation } \\
\hline \multirow{7}{*}{$\begin{array}{l}\text { Null Hypothesis } \\
\mathcal{P}(i \uparrow \mid \pi \downarrow, u \downarrow) \geq \mathcal{P}(i \uparrow \mid \pi \uparrow, u \downarrow) \\
\mathcal{P}(i \uparrow \mid \pi \downarrow, u \uparrow) \geq \mathcal{P}(i \uparrow \mid \pi \uparrow, u \uparrow) \\
\mathcal{P}(i \downarrow \mid \pi \uparrow, u \downarrow) \geq \mathcal{P}(i \downarrow \mid \pi \downarrow, u \downarrow) \\
\mathcal{P}(i \downarrow \mid \pi \uparrow, u \uparrow) \geq \mathcal{P}(i \downarrow \mid \pi \downarrow, u \uparrow)\end{array}$} & \multicolumn{3}{|c|}{$18-29$ years } & \multicolumn{3}{|c|}{$30-45$ years } \\
\hline & mean diff. & test stat. & p-value & mean diff & test stat. & p-value \\
\hline & 0.08 & 3.10 & 0.00 & 0.10 & 5.81 & 0.00 \\
\hline & 0.08 & 4.76 & 0.00 & 0.11 & 10.95 & 0.00 \\
\hline & 0.03 & 1.48 & 0.07 & 0.03 & 2.17 & 0.01 \\
\hline & 0.04 & 4.75 & 0.00 & 0.05 & 7.12 & 0.00 \\
\hline & \multicolumn{3}{|c|}{$46-60$ years } & \multicolumn{3}{|c|}{$60-80$ years } \\
\hline Null Hypothesis & mean diff. & test stat. & p-value & mean diff & test stat. & $\mathrm{p}$-value \\
\hline $\mathcal{P}(i \uparrow \mid \pi \downarrow, u \downarrow) \geq \mathcal{P}(i \uparrow \mid \pi \uparrow, u \downarrow)$ & 0.11 & 6.16 & 0.00 & 0.10 & 5.97 & 0.00 \\
\hline $\mathcal{P}(i \uparrow \mid \pi \downarrow, u \uparrow) \geq \mathcal{P}(i \uparrow \mid \pi \uparrow, u \uparrow)$ & 0.11 & 8.96 & 0.00 & 0.09 & 8.23 & 0.00 \\
\hline $\mathcal{P}(i \downarrow \mid \pi \uparrow, u \downarrow) \geq \mathcal{P}(i \downarrow \mid \pi \downarrow, u \downarrow)$ & 0.03 & 2.35 & 0.01 & 0.03 & 2.66 & 0.00 \\
\hline $\mathcal{P}(i \downarrow \mid \pi \uparrow, u \uparrow) \geq \mathcal{P}(i \downarrow \mid \pi \downarrow, u \uparrow)$ & 0.08 & 8.27 & 0.00 & 0.06 & 5.88 & 0.00 \\
\hline \multicolumn{7}{|c|}{ Partial effects of unemployment } \\
\hline & \multicolumn{3}{|c|}{$18-29$ years } & \multicolumn{3}{|c|}{$30-45$ years } \\
\hline Null Hypothesis & mean diff. & test stat. & p-value & mean diff & test stat. & p-value \\
\hline $\mathcal{P}(i \uparrow \mid \pi \downarrow, u \uparrow) \geq \mathcal{P}(i \uparrow \mid \pi \downarrow, u \downarrow)$ & -0.06 & -1.94 & 0.97 & -0.03 & -2.01 & 0.81 \\
\hline $\mathcal{P}(i \uparrow \mid \pi \uparrow, u \uparrow) \geq \mathcal{P}(i \uparrow \mid \pi \uparrow, u \downarrow)$ & -0.05 & -2.58 & 1.00 & -0.04 & -3.76 & 0.98 \\
\hline $\mathcal{P}(i \downarrow \mid \pi \downarrow, u \downarrow) \geq \mathcal{P}(i \downarrow \mid \pi \downarrow, u \uparrow)$ & 0.03 & 3.60 & 0.00 & 0.04 & 4.07 & 0.00 \\
\hline \multirow{2}{*}{$\mathcal{P}(i \downarrow \mid \pi \uparrow, u \downarrow) \geq \mathcal{P}(i \downarrow \mid \pi \uparrow, u \uparrow)$} & 0.02 & 1.36 & 0.09 & 0.01 & 1.10 & 0.14 \\
\hline & \multicolumn{3}{|c|}{$46-60$ years } & \multicolumn{3}{|c|}{$60-80$ years } \\
\hline Null Hypothesis & mean diff. & test stat. & $\mathrm{p}$-value & mean diff & test stat. & $\mathrm{p}$-value \\
\hline $\mathcal{P}(i \uparrow \mid \pi \downarrow, u \uparrow) \geq \mathcal{P}(i$ & -0.01 & -1.17 & 0.885 & 0.03 & 2.09 & 0.02 \\
\hline $\mathcal{P}(i \uparrow \mid \pi \uparrow, u \uparrow) \geq \mathcal{P}(i \uparrow \mid \pi \uparrow, u \downarrow)$ & -0.02 & -0.73 & 0.77 & 0.05 & 2.84 & 0.00 \\
\hline $\mathcal{P}(i \downarrow \mid \pi \downarrow, u \downarrow) \geq \mathcal{P}(i \downarrow \mid \pi \downarrow, u \uparrow)$ & 0.06 & 5.01 & 0.00 & 0.08 & 7.05 & 0.00 \\
\hline $\mathcal{P}(i \downarrow \mid \pi \uparrow, u \downarrow) \geq \mathcal{P}(i \downarrow \mid \pi \uparrow, u \uparrow)$ & 0.01 & 1.57 & 0.06 & 0.05 & 5.88 & 0.00 \\
\hline
\end{tabular}

Test statistics and p-values are based on Newey-West standard errors with correction for up to 12 lags.

Table 8: Frequencies of responses - SPF (\%)

\begin{tabular}{ccccc}
\hline \hline & \multicolumn{3}{c}{ Unconditional frequencies } & \\
\cline { 2 - 4 } $\mathcal{P}(i)$ & $\downarrow$ & $\leftrightarrow$ & $\uparrow$ & \\
$\mathcal{P}(\pi)$ & 35.05 & - & 64.96 & \\
$\mathcal{P}(u)$ & 47.90 & - & 52.10 & \\
& & \multicolumn{4}{c}{ Conditional frequencies } \\
\cline { 2 - 5 } & $\downarrow$ & $\leftrightarrow$ & $\uparrow$ & $\#$ Obs \\
$\mathcal{P}(i \mid \pi \uparrow, u \downarrow)$ & 16.10 & - & 83.90 & 652 \\
$\mathcal{P}(i \mid \pi \uparrow, u \leftrightarrow)$ & - & - & - & 0 \\
$\mathcal{P}(i \mid \pi \uparrow, u \uparrow)$ & 45.30 & - & 54.70 & 649 \\
$\mathcal{P}(i \mid \pi \downarrow, u \downarrow)$ & 18.91 & - & 81.09 & 476 \\
$\mathcal{P}(i \mid \pi \downarrow, u \leftrightarrow)$ & 25.00 & - & 75.00 & 4 \\
$\mathcal{P}(i \mid \pi \downarrow, u \uparrow)$ & 53.77 & - & 46,23 & 716 \\
\hline \hline
\end{tabular}


by professional forecasters. Perhaps surprisingly, none of the partial effects of inflation implied by the Taylor rule are statistically significant at the usual significance levels. In contrast, the partial effects of unemployment are statistically significant, in line with the Taylor rule, and quite large when compared to the partial effects of unemployment based on the Michigan Survey.

Table 9: Partial effects - SPF

\begin{tabular}{lccc}
\hline \hline \multicolumn{4}{c}{ Partial effects of inflation } \\
\hline Null Hypothesis & mean diff & t-stat & p-value \\
$\mathcal{P}(i \uparrow \mid \pi \downarrow, u \downarrow) \geq \mathcal{P}(i \uparrow \mid \pi \uparrow, u \downarrow)$ & 0.03 & 0.91 & 0.18 \\
$\mathcal{P}(i \uparrow \mid \pi \downarrow, u \uparrow) \geq \mathcal{P}(i \uparrow \mid \pi \uparrow, u \uparrow)$ & 0.08 & 2.46 & 0.01 \\
& & & \\
$\mathcal{P}(i \downarrow \mid \pi \uparrow, u \downarrow) \geq \mathcal{P}(i \downarrow \mid \pi \downarrow, u \downarrow)$ & 0.03 & 0.91 & 0.18 \\
$\mathcal{P}(i \downarrow \mid \pi \uparrow, u \uparrow) \geq \mathcal{P}(i \downarrow \mid \pi \downarrow, u \uparrow)$ & 0.08 & 2.46 & 0.01 \\
\multicolumn{5}{c}{ Partial effects of unemployment } \\
\hline Null Hypothesis $\quad$ mean diff & t-stat & p-value \\
$\mathcal{P}(i \uparrow \mid \pi \downarrow, u \uparrow) \geq \mathcal{P}(i \uparrow \mid \pi \downarrow, u \downarrow)$ & 0.34 & 10.30 & 0.00 \\
$\mathcal{P}(i \uparrow \mid \pi \uparrow, u \uparrow) \geq \mathcal{P}(i \uparrow \mid \pi \uparrow, u \downarrow)$ & 0.29 & 9.69 & 0.00 \\
$\mathcal{P}(i \downarrow \mid \pi \downarrow, u \downarrow) \geq \mathcal{P}(i \downarrow \mid \pi \downarrow, u \uparrow)$ & 0.34 & 10.30 & 0.00 \\
$\mathcal{P}(i \downarrow \mid \pi \uparrow, u \downarrow) \geq \mathcal{P}(i \downarrow \mid \pi \uparrow, u \uparrow)$ & 0.29 & 6.69 & 0.00 \\
\hline \hline Test statistics and p-values are based on Newey-West standard errors with cor- \\
rection for up to 4 lags.
\end{tabular}

The results on the partial effects of inflation and unemployment may suggest that professional forecasters perceive the relationship between interest-rate decisions, inflation, and unemployment to be tilted towards the employment dimension. Alternatively, they may imply a perception that headline inflation is not the most important metric for the FOMC's gauge of price stability. As an attempt to test the latter conjecture, we redo our analysis of the partial effects of inflation perceived by professional forecasters using their forecasts for core CPI (excluding food and energy) instead of headline CPI inflation. We note the caveat that the SPF only started asking participants about their forecasts for core CPI in 2007, so our sample is limited to a single year. Despite this data limitation, Table 10 shows that once the partial effects of inflation are cast in terms of core rather than headline CPI, they become larger, and some of them become statistically significant. This suggests that professional forecasters indeed have a more nuanced view of how monetary policy responds to inflation. ${ }^{15}$

\section{Interpreting our results}

\subsection{Endogeneity and causality}

An issue that we haven't discussed to this point is how to think about endogeneity and causality given our empirical approach. If none of the variation in inflation and unemployment comes from

\footnotetext{
${ }^{15}$ This view was well justified prior to the Fed's recent statement that spells out a measure of headline inflation as being the relevant one to define its goal of price stability. See footnote 4.
} 
Table 10: Partial effects of core inflation - SPF

\begin{tabular}{lccc}
\hline \hline Null Hypothesis & mean diff & t-stat & p-value \\
$\mathcal{P}(i \uparrow \mid \pi \downarrow, u \downarrow) \geq \mathcal{P}(i \uparrow \mid \pi \uparrow, u \downarrow)$ & 0.32 & 2.19 & 0.01 \\
$\mathcal{P}(i \uparrow \mid \pi \downarrow, u \uparrow) \geq \mathcal{P}(i \uparrow \mid \pi \uparrow, u \uparrow)$ & 0.24 & 2.60 & 0.00 \\
& & & \\
$\mathcal{P}(i \downarrow \mid \pi \uparrow, u \downarrow) \geq \mathcal{P}(i \downarrow \mid \pi \downarrow, u \downarrow)$ & 0.32 & 2.19 & 0.01 \\
$\mathcal{P}(i \downarrow \mid \pi \uparrow, u \uparrow) \geq \mathcal{P}(i \downarrow \mid \pi \downarrow, u \uparrow)$ & 0.24 & 2.60 & 0.00 \\
\hline \hline
\end{tabular}

departures from the Fed's systematic interest-rate policy ("monetary policy shocks"), then endogeneity is not a problem. If this is not the case, and such monetary shocks affect the endogenous determination of inflation and output, then there is a clear problem of endogeneity, and our empirical approach need not recover the true causal relationship between inflation and unemployment on one side and interest rates on the other side. So, how do we explore about this issue given that, in reality, there is evidence that interest-rate shocks do affect inflation and economic activity?

Our view is that, given our empirical approach, this is not likely to be a quantitatively important issue. The reasons are twofold. First, most evidence about the effects of monetary (interest-rate) shocks suggests that they only explain a small to moderate fraction of the variance of inflation and unemployment (e.g., Leeper, Sims, and Zha 1996). Second, while any extent of endogeneity bias immediately creates a problem for OLS-based inference about the magnitude of the parameters of the monetary policy rule that control the causal effects of interest, this may still not matter for our conclusions. The reason is that our analysis is based on the signs of the effects of inflation and unemployment on interest rates - not on the magnitude of these effects. Hence, to the extent that the endogeneity bias affects the magnitude of the estimated coefficients in the reduced-form relationship of interest between interest rates, inflation, and unemployment but does not affect their sign, it does not matter for our conclusions.

To test our conjecture, we simulate a new Keynesian DSGE economy and apply our empirical approach to model-generated data. We use the Galí, Smets, and Wouters (2011) estimated DSGE model of the U.S. economy, which includes unemployment. ${ }^{16}$ In their estimated model, shocks to the monetary policy rule explain about $7.6 \%$ of the variance of inflation and $6.5 \%$ of the variance of unemployment. We obtain artificial time series for the policy rate, inflation, and unemployment. For each simulated series, we build categorical variables corresponding to the direction of 12-month changes of each of the three variables, just as we do with realized data. We then apply our empirical approach to draw inferences about the partial effects of inflation and unemployment.

The Taylor rule in the Galí-Smets-Wouters model includes an interest-rate smoothing component, as well as current inflation and unemployment and the change in unemployment. In addition to their model, we estimate a variant with an alternative, simpler Taylor rule whereby interest rates only respond to current unemployment and 4 -quarter inflation. ${ }^{17}$ In this alternative esti-

\footnotetext{
${ }^{16}$ We thank the authors for kindly providing us with their codes.

${ }^{17}$ The model is estimated using the exact same data and Bayesian methods applied by Galí, Smets, and Wouters
} 
mated model, shocks to the monetary policy rule explain about $5.1 \%$ of the variance of inflation and $4.5 \%$ of the variance of unemployment.

We also consider variants of the two estimated models, obtained by increasing the variance of the monetary policy shock relative to the estimated values, while keeping all other estimated parameter values unchanged. With this comparison we want to assess the effects of increasing the degree of endogeneity of inflation and unemployment with respect to policy shocks. Under those two alternative specifications for the Taylor rule, and alternative assumptions for the relative importance of exogenous movements in interest rates, we use the simulated data to calculate the partial effects of inflation and unemployment.

Table 11: Partial effects in the Galí, Smets, and Wouters (2011) model

\begin{tabular}{|c|c|c|c|c|c|c|}
\hline \multicolumn{7}{|c|}{ Partial effects of inflation } \\
\hline & \multicolumn{3}{|c|}{ GSW - baseline } & \multicolumn{3}{|c|}{ GSW - volatile shocks } \\
\hline Null Hypothesis & mean diff. & test stat. & p-value & mean diff. & test stat. & p-value \\
\hline $\mathcal{P}(i \uparrow \mid \pi \downarrow, u \downarrow) \geq \mathcal{P}(i \uparrow \mid \pi \uparrow, u \downarrow)$ & 0.27 & 36.52 & 0.00 & 0.14 & 17.70 & 0.00 \\
\hline $\mathcal{P}(i \uparrow \mid \pi \downarrow, u \uparrow) \geq \mathcal{P}(i \uparrow \mid \pi \uparrow, u \uparrow)$ & 0.27 & 37.08 & 0.00 & 0.14 & 18.06 & 0.00 \\
\hline $\mathcal{P}(i \downarrow \mid \pi \uparrow, u \downarrow) \geq \mathcal{P}(i \downarrow \mid \pi \downarrow, u \downarrow)$ & 0.27 & 36.52 & 0.00 & 0.14 & 17.70 & 0.00 \\
\hline \multirow[t]{2}{*}{$\mathcal{P}(i \downarrow \mid \pi \uparrow, u \uparrow) \geq \mathcal{P}(i \downarrow \mid \pi \downarrow, u \uparrow)$} & 0.27 & 37.08 & 0.00 & 0.14 & 18.06 & 0.00 \\
\hline & \multicolumn{3}{|c|}{ Simple TR - baseline } & \multicolumn{3}{|c|}{ Simple TR - volatile shocks } \\
\hline Null Hypothesis & mean diff. & test stat. & p-value & mean diff. & test stat. & p-value \\
\hline $\mathcal{P}(i \uparrow \mid \pi \downarrow, u \downarrow) \geq \mathcal{P}(i \uparrow \mid \pi \uparrow, u \downarrow)$ & 0.54 & 85.61 & 0.00 & 0.33 & 46.64 & 0.00 \\
\hline $\mathcal{P}(i \uparrow \mid \pi \downarrow, u \uparrow) \geq \mathcal{P}(i \uparrow \mid \pi \uparrow, u \uparrow)$ & 0.54 & 86.07 & 0.00 & 0.33 & 46.49 & 0.00 \\
\hline $\mathcal{P}(i \downarrow \mid \pi \uparrow, u \downarrow) \geq \mathcal{P}(i \downarrow \mid \pi \downarrow, u \downarrow)$ & 0.54 & 85.61 & 0.00 & 0.33 & 46.64 & 0.00 \\
\hline $\mathcal{P}(i \downarrow \mid \pi \uparrow, u \uparrow) \geq \mathcal{P}(i \downarrow \mid \pi \downarrow, u \uparrow)$ & 0.54 & 86.07 & 0.00 & 0.33 & 46.49 & 0.00 \\
\hline \multicolumn{7}{|c|}{ Partial Effects of Unemployment } \\
\hline & \multicolumn{3}{|c|}{ GSW - baseline } & \multicolumn{3}{|c|}{ GSW - volatile shocks } \\
\hline Null Hypothesis & mean diff. & test stat. & p-value & mean diff. & test stat. & p-value \\
\hline $\mathcal{P}(i \uparrow \mid \pi \downarrow, u \downarrow) \geq \mathcal{P}(i \uparrow \mid \pi \uparrow, u \downarrow)$ & 0.29 & 39.93 & 0.00 & -0.17 & -21.63 & 1.00 \\
\hline $\mathcal{P}(i \uparrow \mid \pi \downarrow, u \uparrow) \geq \mathcal{P}(i \uparrow \mid \pi \uparrow, u \uparrow)$ & 0.28 & 38.62 & 0.00 & -0.17 & -22.24 & 1.00 \\
\hline $\mathcal{P}(i \downarrow \mid \pi \uparrow, u \downarrow) \geq \mathcal{P}(i \downarrow \mid \pi \downarrow, u \downarrow)$ & 0.29 & 39.93 & 0.00 & -0.17 & -21.63 & 1.00 \\
\hline \multirow{2}{*}{$\mathcal{P}(i \downarrow \mid \pi \uparrow, u \uparrow) \geq \mathcal{P}(i \downarrow \mid \pi \downarrow, u \uparrow)$} & 0.28 & 38.62 & 0.00 & -0.17 & -22.24 & 1.00 \\
\hline & \multicolumn{3}{|c|}{ Simple TR - baseline } & \multicolumn{3}{|c|}{ Simple TR - volatile shocks } \\
\hline Null Hypothesis & mean diff. & test stat. & p-value & mean diff. & test stat. & p-value \\
\hline $\mathcal{P}(i \uparrow \mid \pi \downarrow, u \uparrow) \geq \mathcal{P}(i \uparrow \mid \pi \downarrow, u \downarrow)$ & 0.17 & 27.66 & 0.00 & -0.16 & -22.46 & 1.00 \\
\hline $\mathcal{P}(i \uparrow \mid \pi \uparrow, u \uparrow) \geq \mathcal{P}(i \uparrow \mid \pi \uparrow, u \downarrow)$ & 0.17 & 26.83 & 0.00 & -0.16 & -22.83 & 1.00 \\
\hline $\mathcal{P}(i \downarrow \mid \pi \downarrow, u \downarrow) \geq \mathcal{P}(i \downarrow \mid \pi \downarrow, u \uparrow)$ & 0.17 & 27.66 & 0.00 & -0.16 & -22.46 & 1.00 \\
\hline $\mathcal{P}(i \downarrow \mid \pi \uparrow, u \downarrow) \geq \mathcal{P}(i \downarrow \mid \pi \uparrow, u \uparrow)$ & 0.17 & 26.83 & 0.00 & -0.16 & -22.83 & 1.00 \\
\hline
\end{tabular}

Test statistics and p-values are based on Newey-West standard errors with correction for up to 4 lags.

Table 11 presents the results. Panels labeled with "GSW" provide the results for the simulated model using the original Galí-Smets-Wouters specification for the Taylor rule, while panels labeled with "Simple TR" provide the results from the model with the alternative Taylor rule. We present results using the estimated parameter values - indicated by the label "baseline" - and results with more volatile monetary policy shocks - indicated by the label "volatile shocks". For the latter we (2011). For brevity we do not provide a detailed explanation of the estimation process here, and refer readers to their paper. 
increase the estimated variance of monetary policy shocks by a factor of ten.

Confirming our intuition, we find that the partial effects of inflation and unemployment obtained from data generated by the estimated models come out with the expected signs, and are statistically significant irrespective of the Taylor rule specification. We also confirm the intuition that our approach to inference might become invalid if monetary policy shocks are more volatile. In particular, with policy shocks that are ten times more volatile, the partial effects of unemployment come out with the wrong sign. This reflects reverse causality running from interest rates to unemployment. With large policy shocks, exogenous movements in interest rates explain a relatively large fraction of the variance of unemployment. An exogenous increase in interest rates induces a decline in unemployment in equilibrium, and this is what produces the inverse sign in the partial effects of unemployment. ${ }^{18}$ However, with more volatile policy shocks, the fraction of the variance of inflation and unemployment that they account for becomes counterfactually large above $30 \%$ for the model with the simple Taylor rule, and above $40 \%$ for the Gali-Smets-Wouters model. We find that our approach to inference works well with monetary shocks that are up to four times more volatile than what the estimated models imply. Beyond that point the partial effects of unemployment start to come out with the wrong sign. ${ }^{19}$

\subsection{Heterogeneity and differences of opinion}

In this section we present a simple framework to interpret our empirical approach. The goal is to have a setting in which we can think formally about how to interpret the partial effects and the statistical tests performed in Section 3.

Our starting point is a population of households, some of which reason according to a simple form of the Taylor rule. We assume that a such household has in mind a simple Taylor rule with two additive components. The first is a "systematic" component that satisfies the stabilization principles underlying the Taylor rule and which dictates how, on average, the household's interestrate forecast for a given date $T$ should be related to the same household's forecasts of inflation and unemployment for the same date. We denote this systematic interest-rate forecast by $i_{t, T}^{*}$, and assume that it only depends on the household's forecasts of unemployment for date $T$ (denoted $u_{t, T}$ ) and of inflation in the 12 -month period ending at date $T$ (denoted $\pi_{t, T}$ ), according to a simple Taylor-type rule: ${ }^{20}$

$$
i_{t, T}^{*}=\phi_{\pi} \pi_{t, T}+\phi_{u} u_{t, T}
$$

The second component of the household's interest-rate forecast for date $T$ is the amount by which the household believes the interest rate will deviate from the systematic forecast $i_{t, T}^{j *}$. We introduce this forecast shock component (denoted $-\varepsilon_{t, T}$ ) because equation (9) is an imperfect description of

\footnotetext{
${ }^{18}$ The same does not occur with inflation. We conjecture that this has to do with the Taylor principle - the fact that the elasticity of the endogenous response of interest rates to inflation is greater than unity.

${ }^{19}$ The fraction of the variance of inflation and unemployment accounted for by monetary policy shocks is also counterfactually large at this threshold level for the variance of monetary shocks: above $16 \%$ in the model with the simple Taylor rule, and above $20 \%$ in the Galí-Smets-Wouters model.

${ }^{20}$ For simplicity we abstract from variations in the unemployment rate consistent with full employment and in the Fed's inflation objective.
} 
how the Fed sets its policy rate, and so we want to allow a household that understands the conduct of monetary policy to make interest-rate forecasts that deviate from its systematic component. We think of $\varepsilon_{t, T}$ as being drawn by the household when forming its interest-rate forecast, from a distribution with cumulative distribution function $F_{\varepsilon}(\varepsilon)$, and assume that $\varepsilon_{t, T}$ is independent of $\pi_{t, T}, u_{t, T}$ and of $i_{t, t}, \pi_{t, t}, u_{t, t}$ - this assumption rules out endogeneity problems, and is justified on the basis of the results of the previous subsection. Our previous assumption that $i_{t, T}^{*}$ is the forecast that the household makes on average implies that $\varepsilon_{t, T}$ has mean zero.

The variables $i_{t, t}, \pi_{t, t}$, and $u_{t, t}$ denote, respectively, the household's perceptions of the policy rate at time $t$, of inflation in the 12 months up to and including time $t$, and of the unemployment rate at time $t$, which we assume to coincide with the actual values of these three variables (i.e., we assume that they are known to the households when they answer the survey questions at time $t$ ). Because we assume $i_{t, t}, \pi_{t, t}$, and $u_{t, t}$ to be known at time $t$, the realization of $\varepsilon_{t, t}$ also becomes known at time $t$.

The actual numerical interest-rate forecast of the household is denoted by $i_{t, T}=i_{t, T}^{*}-\varepsilon_{t, T}$ :

$$
i_{t, T}=\phi_{\pi} \pi_{t, T}+\phi_{u} u_{t, T}-\varepsilon_{t, T}
$$

We do not specify how the household forms inflation and unemployment forecasts and only assume that they are based on some model that produces a joint distribution of these two forecasts for any given horizon. ${ }^{21}$

Recall that with the exception of inflation, the questions in the Michigan Survey that we use in our analysis pertain to the direction of interest rates and unemployment in the subsequent 12 months. So, to move closer to the analysis that we perform with available data, we need to go from (10) to a specification in changes instead of levels. To that end, we subtract (10) with $T=t$ from (10) itself to arrive at:

$$
\Delta i_{t, T}=\phi_{\pi} \Delta \pi_{t, T}+\phi_{u} \Delta u_{t, T}-\varepsilon_{t, T}+\varepsilon_{t, t}
$$

where $\Delta x_{t, T}=x_{t, T}-x_{t, t}$ for $x=i, \pi, u$.

With what we have developed so far, we can ask hypothetical questions such as the following: given numerical forecasts of inflation and unemployment that imply a change in 12-month inflation of $\Delta \pi$ and a change in unemployment of $\Delta u$ twelve months from now, what is the probability that the household will answer that interest rates will go up in the next 12 months? Equation (11) implies that:

$$
\Delta i_{t, T} \geq 0 \Longleftrightarrow \varepsilon_{t, T} \leq \phi_{\pi} \Delta \pi_{t, T}+\phi_{u} \Delta u_{t, T}+\varepsilon_{t, t} .
$$

Thus the answer to the hypothetical question would be:

$$
\begin{aligned}
\operatorname{Pr}\left(\Delta i_{t, T} \geq 0 \mid \Omega_{t}\right) & =\operatorname{Pr}\left(\varepsilon_{t, T} \leq \phi_{\pi} \Delta \pi_{t, T}+\phi_{u} \Delta u_{t, T}+\varepsilon_{t, t}\right) \\
& =F_{\varepsilon}\left(\phi_{\pi} \Delta \pi_{t, T}+\phi_{u} \Delta u_{t, T}+\varepsilon_{t, t}\right),
\end{aligned}
$$

\footnotetext{
${ }^{21}$ Alternative assumptions would also work here. For example, we could assume that the household has in mind some fully specified model of the economy in which equation (10) holds, and that the household reports the modal forecast from the joint distribution for the three variables implied by the equilibrium of the model.
} 
where $\Omega_{t}=\left\{\Delta \pi_{t, T}, \Delta u_{t, T}, i_{t, t}, \pi_{t, t}, u_{t, t}, \phi_{\pi}, \phi_{u}\right\}$, and to avoid cluttering the notation we omit the conditioning variables in the expressions in the right-hand-side of the expressions above.

We can also ask simple questions of comparative statics, such as how that probability changes with the household's inflation and unemployment forecasts:

$$
\begin{aligned}
\frac{\partial F_{\varepsilon}\left(\phi_{\pi} \Delta \pi_{t, T}+\phi_{u} \Delta u_{t, T}+\varepsilon_{t, t}\right)}{\partial \Delta \pi_{t, T}} & =F_{\varepsilon}^{\prime}\left(\phi_{\pi} \Delta \pi_{t, T}+\phi_{u} \Delta u_{t, T}+\varepsilon_{t, t}\right) \phi_{\pi} \lessgtr 0, \\
\frac{\partial F_{\varepsilon}\left(\phi_{\pi} \Delta \pi_{t, T}+\phi_{u} \Delta u_{t, T}+\varepsilon_{t, t}\right)}{\partial \Delta u_{t, T}} & =F_{\varepsilon}^{\prime}\left(\phi_{\pi} \Delta \pi_{t, T}+\phi_{u} \Delta u_{t, T}+\varepsilon_{t, t}\right) \phi_{u} \lessgtr 0,
\end{aligned}
$$

where the signs of the expressions (12) and (13) depend on the signs of $\phi_{\pi}$ and $\phi_{u}$, and thus reflect the stabilization principles underlying the Taylor rule if $\phi_{\pi}>0$ and $\phi_{u}<0$.

The results obtained so far would be useful if we could observe numerical forecasts of households over time. However, in the data we analyze we do not observe the same household more than once, ${ }^{22}$ and we only have numerical forecasts for inflation. Our next step is to construct an environment in which we can make comparative statics statements analogous to (12) and (13) based on one-time answers by a pool households.

Assume that we observe answers from a population of households such as the one we have been modeling so far in this subsection, and that there is heterogeneity in their forecasts of interest rates, inflation, and unemployment, and in their perceptions about the policy parameters $\phi_{\pi}$ and $\phi_{u}$. As a first step, assume that inflation and unemployment forecasts are numerical, but interest-rate forecasts are categorical as in our data (up/down/same).

In the context of our simple framework, one way to model an individual household $j$ 's categorical response for the direction of interest rates is to assume that the answer is conditional on the forecast shock $\varepsilon_{t, T}^{j}$ :

$$
\mathbb{1}\left(\Delta i_{t, T}^{j} \geq 0\right)=\left\{\begin{array}{l}
1 \text { if } \varepsilon_{t, T}^{j} \leq \phi_{\pi}^{j} \Delta \pi_{t, T}^{j}+\phi_{u}^{j} \Delta u_{t, T}^{j}+\varepsilon_{t, t}^{j} \\
0 \text { if } \varepsilon_{t, T}^{j}>\phi_{\pi}^{j} \Delta \pi_{t, T}^{j}+\phi_{u}^{j} \Delta u_{t, T}^{j}+\varepsilon_{t, t}^{j}
\end{array} .\right.
$$

Under the additional assumption that the forecast shocks $\varepsilon_{t, T}^{j}$ are independent across households, we can do comparative statics exercises analogous to (12) and (13) by pooling households according to their forecasts of inflation and unemployment. ${ }^{23}$

As an intermediate step, if we select a large pool of households with the same Taylor rule coefficients $\phi_{\pi}, \phi_{u}$, and the same given forecasts for changes in inflation and unemployment $\Delta \pi$ and $\Delta u$, by the law of large numbers the fraction of households answering that interest rates will move

\footnotetext{
${ }^{22}$ As mentioned in Subsection 2.1, the Michigan Survey does have a small panel dimension, since a fraction of households are interviewed a second time. Nevertheless, having at most two observations for some households would not be enough do the empirical counterpart of the comparative static analysis implied by (12) and (13), household by household.

${ }^{23}$ We also extend the assumption of independence between forecast shocks $\varepsilon_{t, T}^{j}$ and $\pi_{t, T}^{j}, u_{t, T}^{j}$ and $i_{t, t}^{j}, \pi_{t, t}^{j}, u_{t, t}^{j}$ for all $j$.
} 
up will be given by: ${ }^{24}$

$$
\begin{aligned}
& \operatorname{Fr}\left(\Delta i \geq 0 \mid \phi_{\pi}, \phi_{u}, \Delta \pi, \Delta u\right)= \\
= & \frac{\int_{j \in J} \mathbb{1}\left(\varepsilon^{j} \leq \phi_{\pi} \Delta \pi+\phi_{u} \Delta u+\varepsilon_{\phi_{\pi}, \phi_{u}}\right) d j}{\int_{j \in J} \mathbb{1}\left(\varepsilon^{j} \leq \phi_{\pi} \Delta \pi+\phi_{u} \Delta u+\varepsilon_{\phi_{\pi}, \phi_{u}}\right) d j+\int_{j \in J} \mathbb{1}\left(\varepsilon^{j}>\phi_{\pi} \Delta \pi+\phi_{u} \Delta u+\varepsilon_{\phi_{\pi}, \phi_{u}}\right) d j} \\
= & F_{\varepsilon}\left(\phi_{\pi} \Delta \pi+\phi_{u} \Delta u+\varepsilon_{\phi_{\pi}, \phi_{u}}\right),
\end{aligned}
$$

where $J$ collects the indices of households in the pool that forecasts $(\Delta \pi, \Delta u)$ and perceives Taylor rule coefficients $\left(\phi_{\pi}, \phi_{u}\right)$. The numerator in equation (14) counts households in that pool who respond that interest rates will increase, and the denominator accounts for all possible responses about future interest rates (i.e., the size of the pool of households in $J$ ). $\varepsilon_{\phi_{\pi}, \phi_{u}}$ represents the perceived policy shock realized at time $t$ as a function of $\phi_{\pi}, \phi_{u} \cdot{ }^{25}$

To obtain the fraction of households that respond that interest rates will increase for given forecasts $\Delta \pi$ and $\Delta u$, we need to integrate over the distribution of policy coefficients $\phi=\left(\phi_{\pi}, \phi_{u}\right)$, which we denote by $F_{\phi}\left(\phi_{\pi}, \phi_{u}\right):{ }^{26}$

$$
\operatorname{Fr}(\Delta i \geq 0 \mid \Delta \pi, \Delta u)=\int_{\phi} F_{\varepsilon}\left(\phi_{\pi} \Delta \pi+\phi_{u} \Delta u+\varepsilon_{\phi_{\pi}, \phi_{u}}\right) d F_{\phi}\left(\phi_{\pi}, \phi_{u}\right) .
$$

If we select another large pool of households with the same forecasted change in unemployment $\Delta u$, but with a higher forecast for the change in inflation $\left(\Delta \pi+\delta_{\pi}\right)$, the difference between the two pools in the fraction of households answering that interest rates will go up will be given by:

$$
\int_{\phi}\left[F_{\varepsilon}\left(\phi_{\pi}\left(\Delta \pi+\delta_{\pi}\right)+\phi_{u} \Delta u+\varepsilon_{\phi_{\pi}, \phi_{u}}\right)-F_{\varepsilon}\left(\phi_{\pi} \Delta \pi+\phi_{u} \Delta u+\varepsilon_{\phi_{\pi}, \phi_{u}}\right)\right] d F_{\phi}\left(\phi_{\pi}, \phi_{u}\right) .
$$

We refer to quantities such as (15) as the $\delta_{\pi}$-partial effects of inflation. Dividing (15) by $\delta_{\pi}$ and taking the limit as $\delta_{\pi} \rightarrow 0$ yields the comparative statics result analogous to (12), i.e.:

$$
\begin{aligned}
& \lim _{\delta_{\pi} \rightarrow 0} \frac{\int_{\phi}\left[F_{\varepsilon}\left(\phi_{\pi}\left(\Delta \pi+\delta_{\pi}\right)+\phi_{u} \Delta u+\varepsilon_{\phi_{\pi}, \phi_{u}}\right)-F_{\varepsilon}\left(\phi_{\pi} \Delta \pi+\phi_{u} \Delta u+\varepsilon_{\phi_{\pi}, \phi_{u}}\right)\right] d F_{\phi}\left(\phi_{\pi}, \phi_{u}\right)}{\delta_{\pi}} \\
= & \int_{\phi} F_{\varepsilon}^{\prime}\left(\phi_{\pi} \Delta \pi+\phi_{u} \Delta u+\varepsilon_{\phi_{\pi}, \phi_{u}}\right) \phi_{\pi} d F_{\phi}\left(\phi_{\pi}, \phi_{u}\right)
\end{aligned}
$$

Likewise, we can compute the $\delta_{u}$-partial effects of unemployment by appropriate choice of two pools of households that share the same forecast for the change in inflation, but differ in the forecast for the change in unemployment (by $\delta_{u}$ ). The difference between the fractions of households

\footnotetext{
${ }^{24}$ To avoid cluttering the notation, from now on we drop the time subscripts.

${ }^{25}$ This depends on the Taylor rule coefficients because it is assumed to be inferred from the observation of the realized values for the interest rate, inflation, and unemployment.

${ }^{26}$ We assume that $\phi$ is distributed independently of all other variables in the population of households.
} 
answering that interest rates will go up in the two pools will be given by:

$$
\int_{\phi}\left[F_{\varepsilon}\left(\phi_{\pi} \Delta \pi+\phi_{u}\left(\Delta u+\delta_{u}\right)+\varepsilon_{\phi_{\pi}, \phi_{u}}\right)-F_{\varepsilon}\left(\phi_{\pi} \Delta \pi+\phi_{u} \Delta u+\varepsilon_{\phi_{\pi}, \phi_{u}}\right)\right] d F_{\phi}\left(\phi_{\pi}, \phi_{u}\right) .
$$

The comparative statics result analogous to that in equation (13) can be obtained as before, dividing equation (17) by $\delta_{u}$ and taking the limit as $\delta_{u} \rightarrow 0$.

Hence, we can write the partial effects of inflation and unemployment as:

$$
\begin{aligned}
& \frac{\partial F r(\Delta i \geq 0 \mid \Delta \pi, \Delta u)}{\partial \Delta \pi}=\int_{\phi} F_{\varepsilon}^{\prime}\left(\phi_{\pi} \Delta \pi+\phi_{u} \Delta u+\varepsilon_{\phi_{\pi}, \phi_{u}}\right) \phi_{\pi} d F_{\phi}\left(\phi_{\pi}, \phi_{u}\right), \\
& \frac{\partial F r(\Delta i \geq 0 \mid \Delta \pi, \Delta u)}{\partial \Delta u}=\int_{\phi} F_{\varepsilon}^{\prime}\left(\phi_{\pi} \Delta \pi+\phi_{u} \Delta u+\varepsilon_{\phi_{\pi}, \phi_{u}}\right) \phi_{u} d F_{\phi}\left(\phi_{\pi}, \phi_{u}\right) .
\end{aligned}
$$

Under the special case where $\varepsilon$ has a uniform distribution, $F_{\varepsilon}^{\prime}$ is a constant and the partial effects above are proportional to the average values of $\phi_{\pi}$ and $\phi_{u}$ in the population. More generally, the partial effects uncover weighted averages of the Taylor rule coefficients in the population of respondents. This result motivates the way we interpret our estimated partial effects, as being informative of the perceptions that some households have about monetary policy.

Finally, note that we have assumed that the forecasts for inflation and unemployment are numerical, whereas in our baseline results we use categorical answers for the questions about the future path of these two variables. Conditioning on the direction of the forecasted change instead of on the numerical forecasts can make a difference for some of the results derived in the model. For instance, it may cause answers that were otherwise well defined to depend on the distribution of Taylor rule coefficients perceived by households in the pool of respondents.

Since we only have categorical responses regarding the future path of unemployment, our empirical approach for estimating the partial effects of inflation can only be imperfectly justified on the basis of the framework developed in this section. We could try to condition on households' answers to other questions in the Michigan Survey, in the hope that doing so would make it more likely that the selected pool of respondents had in mind the same (unobserved) forecasted change in unemployment when answering the survey question. However, we would still have to live with the possibility that unobserved heterogeneity in those numerical forecasts would cause problems.

Fortunately, as described in Subsection 2.1, the Michigan Survey does ask households about their numerical forecasts for inflation. In the Appendix we present robustness results for the partial effects of unemployment using those numerical inflation forecasts - instead of only the direction for the change in inflation. In particular, we test for the partial effects of unemployment by deciles of inflation forecasts. The results show that the substantive conclusions that can be drawn in this case are the same that can be drawn from our analysis in Section 3. 


\subsection{An important identifying assumption}

Our simple framework helps us think about how a household that reasons in accordance with the stabilization principles underlying the Taylor rule might answer categorical questions about interest rates, such as the one in the Michigan Survey. It also shows how, within the framework, those principles can be recovered from answers by a pool of such households that differ in their forecasts of inflation and unemployment, and also possibly in their perceived Taylor rule coefficients.

It is natural to ask whether the lessons we can draw from our analysis are robust to alternative assumptions, even strictly within our simple framework. An important identifying assumption that we make is that households' answers about the direction of interest rates are indeed conditional on their responses about inflation and unemployment (which we assume come from an unspecified model that produces a joint forecast for the path of these two variables). If that is not the case and households do not answer conditionally, one can build examples where a respondent thinks in accordance with the Taylor rule but answers in a way that does not reflect so. ${ }^{27}$

For example, consider a household that forms its interest-rate expectations according to the Taylor rule but answers the survey questionnaire by providing the modal forecast from the marginal distributions of the three variables. For simplicity, assume that the joint probability distributions for inflation and unemployment are such that:

$$
\left.\begin{array}{l}
P(\pi \uparrow, u \downarrow)=0.5 \\
P(\pi \downarrow, u \uparrow)=0.5 \\
P(\pi \uparrow, u \uparrow)=0.0 \\
P(\pi \downarrow, u \downarrow)=0.0
\end{array}\right\} \Longrightarrow \begin{aligned}
& P(\pi \uparrow)=P(\pi \downarrow)=0.5 \\
& P(u \uparrow)=P(u \downarrow)=0.5
\end{aligned} .
$$

Since the household reasons according to the Taylor rule, this implies that $P(i \uparrow)=P(i \downarrow)=0.5$.

If the household answers the survey questions by first making a joint forecast for inflation and unemployment, and then computing the implied interest-rate movement (conditional on that forecast), then $P(i \uparrow \mid \pi \uparrow, u \downarrow)=1$ and $P(i \downarrow \mid \pi \downarrow, u \uparrow)=1$, which is consistent with the stabilization principles underlying the Taylor rule. Likewise, if the household's answers correspond to a point in the joint distribution of interest rates, inflation, and unemployment, then if interestrate expectations accord with the Taylor rule the answers will reveal so. In contrast, if the household answers the questionnaire using the unconditional (marginal) distributions for the three variables separately, the resulting answers could be $\pi \uparrow, u \downarrow$ and $i \downarrow$, which would clearly violate the Taylor rule.

The above example illustrates a more general problem that arises when making inference based on survey data. It has to do precisely with the interpretation of what the responses pertain to. For example, Manski (2005) and Manski and Tamer (2002) raise issues related to those that we face here, in the context of empirical analyses that rely on categorical and interval survey data. Hence, the assumption that households' answers about interest rates are conditional on their answers about inflation and unemployment can be seen as an identifying assumption without which we

\footnotetext{
${ }^{27}$ We thank John Geweke for suggesting this route to highlighting the importance of our conditionality assumption.
} 
could not proceed. In future research, it would be interesting to test this assumption by explicitly asking households about their interest-rate forecasts conditional on their forecasts for inflation and unemployment.

\section{Conclusion}

We combine questions from the Michigan Survey about the future path of prices, interest rates, and unemployment to investigate whether U.S. households are aware of how monetary policy is conducted in the United States. For comparison, we perform the same analysis using questions from the Survey of Professional Forecasters.

Our findings are broadly consistent with the view that some U.S. households are aware of how the Fed conducts monetary policy when forming their expectations about the future paths of interest rates, inflation, and unemployment. The degree of awareness, however, does not appear to be uniform across income and education levels, and age groups. Higher income, more educated, and older households appear to be more aware of the Taylor rule than younger, less educated, and lower income households. In addition, even among the former group of households, people seem to understand the relation between unemployment and interest rates only when it comes to interest rate decreases.

Given the importance that policymakers attach to communicating policy objectives to the public as a way to enhance its effectiveness, we believe that more research should be directed at the more basic question of whether economic agents make sense of the conduct of policy. Our results suggest that there is scope for improving the public's understanding of even some basic dimensions of monetary policy in the United States. Efforts in this direction may improve the effects of the Fed's communication strategy. 


\section{References}

[1] Bernanke, Ben (2007), Federal Reserve Board's semiannual Monetary Policy Report to the Congress, testimony before the Committee on Banking, Housing, and Urban Affairs, US Senate, Washington.

$[2]$ (2010a), "Monetary Policy Objectives and Tools in a Low-Inflation Environment," Speech at the Federal Reserve Bank of Boston (Revisiting Monetary Policy in a LowInflation Environment Conference).

[3] (2010b), "Monetary Policy and the Housing Bubble," Speech at the Annual Meeting of the American Economic Association, Atlanta, Georgia.

[4] Carvalho, F. and A. Minella (2009), "Market Forecasts in Brazil: performance and determinants," Brazilian Central Bank Working Paper Series \# 185.

[5] Eusepi, S. and B. Preston (2010), "Central Bank Communication and Expectations Stabilization," American Economic Journal: Macroeconomics 2: 235-71.

[6] Fendel R., M. Frenkel, and J. Rülke (2011), "'Ex-ante' Taylor rules - Newly discovered evidence from the G7 countries," Journal of Macroeconomics 33: 224-232.

[7] Galí, J., F. Smets, and R. Wouters (2011), "Unemployment in an Estimated New Keynesian Model," NBER Working Papers 17084.

[8] Hamalainen, N. (2004), "A Survey of Taylor-Type Monetary Policy Rules," Canadian Department of Finance Working Paper 2004-02.

[9] Hamilton, J., S. Pruitt, and S. Borger (2011), "Estimating the market-perceived monetary policy rule," American Economic Journal: Macroeconomics 3: 1-28.

[10] Judd, J. and G. Rudebusch (1998), "Taylor's Rule and the Fed: 1970-1997," FRBSF Economic Review, \# 3.

[11] Leeper, E., C. Sims, and T. Zha (1996), "What Does Monetary Policy Do?," Brookings Papers on Economic Activity 27: 1-78.

[12] Manski, C. (2005), "Random Utility Models with Bounded Ambiguity," in Structural Econometrics, Essays in Methodology and Applications, New Delhi: Oxford University Press, 2010, pp. $272-284$.

[13] Manski, C. and E. Tamer (2002), "Inference on Regressions with Interval Data on a Regressor or Outcome," Econometrica 70: 519-546.

[14] Mitchell, K. and D. Pearce (2009), "Do Wall Street economists believe in Okun's Law and the Taylor Rule?" Journal of Economics and Finance 34: 196-217. 
[15] Pesenti, P. (2008), "Comment on 'Reflections on Monetary Policy in the Open Economy'," NBER International Seminar on Macroeconomics.

[16] Schmidt, S. and D. Nautz (2010), "Why Do Financial Market Experts Misperceive Future Monetary Policy Decisions?" SFB 649 Discussion Paper 2010-036.

[17] Taylor, J. (1993), "Discretion versus policy rules in practice," Carnegie-Rochester Conference Series on Public Policy 39: 195-214.

[18] Tortorice, D. (2011), "Unemployment Expectations and the Business Cycle," mimeo.

[19] Williams, J. (2011), "Maintaining Price Stability in a Global Economy," Presentation to Town Hall Los Angeles. 


\section{Appendix}

\subsection{Robustness analysis}

\subsubsection{Information about borrowing rates}

As mentioned previously, the question about interest rates in the Michigan Survey pertains to households' expectations about future borrowing rates. In our benchmark results we assume that the same answers would apply to expectations about the direction of policy rates. Here we redo our analysis of the partial effects of inflation and unemployment restricting the sample to 12-month periods in which borrowing rates and the policy rate moved in the same direction. We consider the 3 -month Treasury bill rate as the policy rate, which is the measure that features in the question of the SPF that we use in our analysis. ${ }^{28}$ As a proxy for borrowing rates, we consider the Freddie Mac national mortgage rate. ${ }^{29}$ Results based on this restricted sample are essentially unchanged. This is the case for both the analysis that pools all household answers - Table 12 - and for the findings based on answers by demographic groups - Table 13.

Table 12: Partial effects of inflation accounting for movements in borrowing rates - Michigan Survey

\begin{tabular}{|c|c|c|c|}
\hline \multicolumn{4}{|c|}{ Partial effects of inflation } \\
\hline Null Hypothesis & mean diff. & test stat. & p-value \\
\hline $\mathcal{P}(i \uparrow \mid \pi \downarrow, u \downarrow) \geq \mathcal{P}(i \uparrow \mid \pi \uparrow, u \downarrow)$ & 0.10 & 9.06 & 0.00 \\
\hline $\mathcal{P}(i \uparrow \mid \pi \downarrow, u \uparrow) \geq \mathcal{P}(i \uparrow \mid \pi \uparrow, u \uparrow)$ & 0.10 & 13.76 & 0.00 \\
\hline $\mathcal{P}(i \downarrow \mid \pi \uparrow, u \downarrow) \geq \mathcal{P}(i \downarrow \mid \pi \downarrow, u \downarrow)$ & 0.03 & 3.42 & 0.00 \\
\hline $\mathcal{P}(i \downarrow \mid \pi \uparrow, u \uparrow) \geq \mathcal{P}(i \downarrow \mid \pi \downarrow, u \uparrow)$ & 0.06 & 11.04 & 0.00 \\
\hline \multicolumn{4}{|c|}{ Partial effects of unemployment } \\
\hline Null Hypothesis & mean diff. & test stat. & p-value \\
\hline $\mathcal{P}(i \uparrow \mid \pi \downarrow, u \uparrow) \geq \mathcal{P}(i \uparrow \mid \pi \downarrow, u \downarrow)$ & -0.01 & -0.80 & 0.79 \\
\hline $\mathcal{P}(i \uparrow \mid \pi \uparrow, u \uparrow) \geq \mathcal{P}(i \uparrow \mid \pi \uparrow, u \downarrow)$ & -0.01 & -0.96 & 0.83 \\
\hline $\mathcal{P}(i \downarrow \mid \pi \downarrow, u \downarrow) \geq \mathcal{P}(i \downarrow \mid \pi \downarrow, u \uparrow)$ & 0.06 & 9.20 & 0.00 \\
\hline $\mathcal{P}(i \downarrow \mid \pi \uparrow, u \downarrow) \geq \mathcal{P}(i \downarrow \mid \pi \uparrow, u \uparrow)$ & 0.03 & 4.63 & 0.04 \\
\hline
\end{tabular}

Test statistics and p-values are based on Newey-West standard errors with correction for up to 12 lags.

\subsubsection{Inflation deciles}

For questions referring to unemployment and interest rates, the Michigan Survey only asks about the direction of change. However, it asks about household inflation expectations. We can thus reestimate the partial effects of unemployment while holding fixed the expected change in inflation. To that end, we divide the expected change in inflation into ten deciles, and estimate the partial

\footnotetext{
${ }^{28}$ Results are robust to using the effective federal funds rate.

${ }^{29}$ Freddie Mac's national mortgage rates are the average of 125 lenders' rates who contributed rates to Freddie Mac. These rates are based on a 30-year fixed-rate mortgage, with $20 \%$ downpayment and $80 \%$ financed over the life of the loan.
} 
Table 13: Partial effects accounting for movements in borrowing rates by income and education

\begin{tabular}{lccccccc}
\hline \hline & \multicolumn{3}{c}{ Partial effects of inflation } & & & \\
\hline & \multicolumn{2}{c}{ Lowest income quartile } & & \multicolumn{2}{c}{ No high school diploma } \\
\cline { 2 - 4 } \cline { 6 - 8 } Null Hypothesis & mean diff. & test stat. & p-value & & mean diff. & test stat. & p-value \\
$\mathcal{P}(i \uparrow \mid \pi \downarrow, u \downarrow) \geq \mathcal{P}(i \uparrow \mid \pi \uparrow, u \downarrow)$ & 0.13 & 5.51 & 0.00 & & 0.14 & 3.75 & 0.00 \\
$\mathcal{P}(i \uparrow \mid \pi \downarrow, u \uparrow) \geq \mathcal{P}(i \uparrow \mid \pi \uparrow, u \uparrow)$ & 0.09 & 6.27 & 0.00 & & 0.10 & 4.24 & 0.00 \\
$\mathcal{P}(i \downarrow \mid \pi \uparrow, u \downarrow) \geq \mathcal{P}(i \downarrow \mid \pi \downarrow, u \downarrow)$ & 0.04 & 2.50 & 0.01 & & 0.07 & 2.36 & 0.01 \\
$\mathcal{P}(i \downarrow \mid \pi \uparrow, u \uparrow) \geq \mathcal{P}(i \downarrow \mid \pi \downarrow, u \uparrow)$ & 0.06 & 5.11 & 0.00 & & 0.05 & 3.99 & 0.00
\end{tabular}

Null Hypothesis

Highest income quartile

$\mathcal{P}(i \uparrow \mid \pi \downarrow, u \downarrow) \geq \mathcal{P}(i \uparrow \mid \pi \uparrow, u \downarrow)$ mean diff. test stat. p-value

At least college degree

$\mathcal{P}(i \uparrow \mid \pi \downarrow, u \uparrow) \geq \mathcal{P}(i \uparrow \mid \pi \uparrow, u \uparrow)$

$\begin{array}{lll}0.08 & 3.57 & 0.00\end{array}$

mean diff. $\quad$ test stat. p-value

$\mathcal{P}(i \downarrow \mid \pi \uparrow, u \downarrow) \geq \mathcal{P}(i \downarrow \mid \pi \downarrow, u \downarrow)$

$\begin{array}{lll}0.11 & 8.35 & 0.00\end{array}$

$\begin{array}{lll}0.09 & 4.96 & 0.00\end{array}$

$0.02 \quad 1.11 \quad 0.13$

$\begin{array}{lll}0.11 & 9.31 & 0.00\end{array}$

$\mathcal{P}(i \downarrow \mid \pi \uparrow, u \uparrow) \geq \mathcal{P}(i \downarrow \mid \pi \downarrow, u \uparrow)$

$\begin{array}{lll}0.07 & 6.89 & 0.00\end{array}$

0.02

$1.57 \quad 0.06$

Partial effects of unemployment

\begin{tabular}{|c|c|c|c|c|c|c|}
\hline \multirow{6}{*}{$\begin{array}{l}\text { Null Hypothesis } \\
\mathcal{P}(i \uparrow \mid \pi \downarrow, u \uparrow) \geq \mathcal{P}(i \uparrow \mid \pi \downarrow, u \downarrow) \\
\mathcal{P}(i \uparrow \mid \pi \uparrow, u \uparrow) \geq \mathcal{P}(i \uparrow \mid \pi \uparrow, u \downarrow) \\
\mathcal{P}(i \downarrow \mid \pi \downarrow, u \downarrow) \geq \mathcal{P}(i \downarrow \mid \pi \downarrow, u \uparrow) \\
\mathcal{P}(i \downarrow \mid \pi \uparrow, u \downarrow) \geq \mathcal{P}(i \downarrow \mid \pi \uparrow, u \uparrow)\end{array}$} & \multicolumn{3}{|c|}{ Lowest income quartile } & \multicolumn{3}{|c|}{ No high school diploma } \\
\hline & mean diff. & test stat. & p-value & -mean diff. & test stat. & p-value \\
\hline & -0.08 & -3.53 & 1.00 & -0.05 & -1.56 & 0.94 \\
\hline & -0.04 & -1.83 & 0.97 & -0.01 & -0.28 & 0.61 \\
\hline & 0.03 & 1.96 & 0.02 & 0.00 & 0.48 & 0.32 \\
\hline & 0.01 & 1.23 & 0.11 & 0.02 & 1.17 & 0.12 \\
\hline & \multicolumn{3}{|c|}{ Highest income quartile } & \multicolumn{3}{|c|}{ At least college degree } \\
\hline Null Hypothesis & mean diff. & test stat. & p-value & mean diff. & test stat. & p-value \\
\hline $\mathcal{P}(i \uparrow \mid \pi \downarrow, u \uparrow) \geq \mathcal{P}(i \uparrow \mid \pi \downarrow, u \downarrow)$ & 0.05 & 3.74 & 0.05 & 0.03 & 2.14 & 0.02 \\
\hline $\mathcal{P}(i \uparrow \mid \pi \uparrow, u \uparrow) \geq \mathcal{P}(i \uparrow \mid \pi \uparrow, u \downarrow)$ & 0.02 & 1.31 & 0.10 & 0.01 & 0.72 & 0.24 \\
\hline $\mathcal{P}(i \downarrow \mid \pi \downarrow, u \downarrow) \geq \mathcal{P}(i \downarrow \mid \pi \downarrow, u \uparrow)$ & 0.10 & 8.67 & 0.00 & 0.10 & 9.36 & 0.00 \\
\hline $\mathcal{P}(i \downarrow \mid \pi \uparrow, u \downarrow) \geq \mathcal{P}(i \downarrow \mid \pi \uparrow, u \uparrow)$ & 0.04 & 4.08 & 0.02 & 0.04 & 4.52 & 0.00 \\
\hline
\end{tabular}

Test statistics and p-values are based on Newey-West standard errors with correction for up to 12 lags. 
effects of unemployment for each decile. While the results vary across deciles, as a general statement they confirm our baseline results (see Table 14). When conditioning on demographics, we find once again that answers by households that have at least a college degree and households at the highest income quartile are more in line with the relationship between unemployment and interest rates implied by the stabilization principles underlying the Taylor rule than answers by households with no high school diploma and by households at the lowest income quartile. For brevity we do not include the corresponding tables, which are available upon request.

Table 14: Partial effects of unemployment by inflation deciles

\begin{tabular}{|c|c|c|c|c|c|c|}
\hline \multirow{5}{*}{$\begin{array}{l}\text { Null Hypothesis } \\
\mathcal{P}(i \uparrow \mid \pi, u \uparrow) \geq \mathcal{P}(i \uparrow \mid \pi, u \downarrow) \\
\mathcal{P}(i \downarrow \mid \pi, u \downarrow) \geq \mathcal{P}(i \downarrow \mid \pi, u \uparrow)\end{array}$} & \multicolumn{3}{|c|}{ decile 1} & \multicolumn{3}{|c|}{ decile 2} \\
\hline & \multirow{4}{*}{$\begin{array}{c}\text { mean diff. } \\
0.03 \\
0.08\end{array}$} & \multirow{4}{*}{$\begin{array}{c}\text { test stat. } \\
0.49 \\
2.80 \\
\text { decile } 3\end{array}$} & \multirow{4}{*}{$\begin{array}{c}\text { p-value } \\
0.31 \\
0.00\end{array}$} & mean diff. & \multirow{4}{*}{$\begin{array}{c}\text { test stat. } \\
1.67 \\
3.86 \\
\text { decile } 4 \\
\end{array}$} & \multirow{4}{*}{$\begin{array}{c}\text { p-value } \\
0.05 \\
0.00\end{array}$} \\
\hline & & & & 0.05 & & \\
\hline & & & & 0.05 & & \\
\hline & & & & & & \\
\hline \multirow{4}{*}{$\begin{array}{l}\text { Null Hypothesis } \\
\mathcal{P}(i \uparrow \mid \pi, u \uparrow) \geq \mathcal{P}(i \uparrow \mid \pi, u \downarrow) \\
\mathcal{P}(i \downarrow \mid \pi, u \downarrow) \geq \mathcal{P}(i \downarrow \mid \pi, u \uparrow)\end{array}$} & \multirow{4}{*}{$\begin{array}{c}\text { mean diff. } \\
0.02 \\
0.05\end{array}$} & \multirow{4}{*}{\multicolumn{2}{|c|}{$\begin{array}{c}\text { test stat. } \\
1.43 \\
4.60 \\
\text { decile } 5 \\
\end{array}$}} & mean diff. & \multirow{4}{*}{$\begin{array}{c}\text { test stat. } \\
0.65 \\
4.34 \\
\text { decile } 6\end{array}$} & \multirow{4}{*}{$\begin{array}{c}\mathrm{p} \text {-value } \\
0.26 \\
0.00\end{array}$} \\
\hline & & & & 0.02 & & \\
\hline & & & & 0.05 & & \\
\hline & & & & & & \\
\hline Null Hypothesis & \multirow{4}{*}{$\begin{array}{c}\text { mean diff. } \\
-0.03 \\
0.07\end{array}$} & \multirow{4}{*}{$\begin{array}{c}\text { test stat. } \\
-1.94 \\
7.29 \\
\text { decile } 7\end{array}$} & \multirow{4}{*}{$\begin{array}{c}\text { p-value } \\
0.97 \\
0.00\end{array}$} & mean diff. & \multirow{4}{*}{$\begin{array}{c}\text { test stat. } \\
0.62 \\
2.35 \\
\text { decile } 8\end{array}$} & \multirow{4}{*}{$\begin{array}{c}\text { p-value } \\
0.27 \\
0.01\end{array}$} \\
\hline $\mathcal{P}(i \uparrow \mid \pi, u \uparrow) \geq \mathcal{P}(i \uparrow \mid \pi, u \downarrow)$ & & & & 0.01 & & \\
\hline $\mathcal{P}(i \downarrow \mid \pi, u \downarrow) \geq \mathcal{P}(i \downarrow \mid \pi, u \uparrow)$ & & & & 0.02 & & \\
\hline & & & & & & \\
\hline Null Hypothesis & \multirow{4}{*}{$\begin{array}{c}\text { mean diff. } \\
0.01 \\
0.01\end{array}$} & \multirow{4}{*}{$\begin{array}{c}\text { test stat. } \\
1.00 \\
2.34 \\
\text { decile } 9 \\
\end{array}$} & \multirow{4}{*}{$\begin{array}{c}\text { p-value } \\
0.16 \\
0.01\end{array}$} & mean diff. & \multirow{4}{*}{$\begin{array}{c}\text { test stat. } \\
-0.12 \\
0.96 \\
\text { decile } 10\end{array}$} & \multirow{4}{*}{$\begin{array}{c}\text { p-value } \\
0.55 \\
0.17\end{array}$} \\
\hline $\mathcal{P}(i \uparrow \mid \pi, u \uparrow) \geq \mathcal{P}(i \uparrow \mid \pi, u \downarrow)$ & & & & -0.01 & & \\
\hline $\mathcal{P}(i \downarrow \mid \pi, u \downarrow) \geq \mathcal{P}(i \downarrow \mid \pi, u \uparrow)$ & & & & 0.01 & & \\
\hline & & & & & & \\
\hline Null Hypothesis & \multirow{3}{*}{$\begin{array}{c}\text { mean diff. } \\
0.04 \\
0.03\end{array}$} & \multirow{3}{*}{$\begin{array}{c}\text { test stat. } \\
2.56 \\
2.30 \\
\end{array}$} & \multirow{3}{*}{$\begin{array}{c}\text { p-value } \\
0.01 \\
0.01\end{array}$} & mean diff. & \multirow{3}{*}{$\begin{array}{l}\text { test stat. } \\
0.76 \\
1.56\end{array}$} & \multirow{3}{*}{$\begin{array}{c}\text { p-value } \\
0.22 \\
0.06\end{array}$} \\
\hline $\mathcal{P}(i \uparrow \mid \pi, u \uparrow) \geq \mathcal{P}(i \uparrow \mid \pi, u \downarrow)$ & & & & 0.02 & & \\
\hline $\mathcal{P}(i \downarrow \mid \pi, u \downarrow) \geq \mathcal{P}(i \downarrow \mid \pi, u \uparrow)$ & & & & 0.02 & & \\
\hline
\end{tabular}

Test statistics and p-values are based on Newey-West standard errors with correction for up to 12 lags.

\subsubsection{Additional robustness analyses ${ }^{30}$}

We did a series of additional robustness analyses. We split our sample between recession and nonrecession periods, ${ }^{31}$ and did separate estimations for each subsample. Households' answers are more in line with the stabilization principles underlying the Taylor rule during non-recession years. An important caveat is that the number of observations for non-recession periods is much higher than for recession periods, so this can be a driver of the results. Nevertheless, for both non-recession and recession periods, answers by households with a college degree and those in the highest income quartile appear to be more in line with the principles underlying the Taylor rule than answers by households with no high school diploma and by those in the lowest income quartile.

We also looked at the partial effects of inflation and unemployment by country regions, and reconfirmed most of our findings. A noteworthy result is that, when it comes to partial effects of

\footnotetext{
${ }^{30}$ All results described in this subsection are available upon request.

${ }^{31}$ We use the NBER recession dates.
} 
unemployment, answers by households in the Northeast are more consistent with the principles underlying the Taylor than answers by households elsewhere in the country.

Throughout the paper we report test statistics and p-values obtained through Newey West corretion for up to 12 lags. We redid all results with windows varying from 1 lag to 12 lags, and our substantive findings are unchanged. In addition, as a way to avoid potential problems generated by the 12-month rolling windows, we redid our analysis using only answers for the same month in each year (from January to December). Our results are again qualitatively unchanged. Interestingly, answers from the January surveys appear to be consistent with the stabilization principles underlying the Taylor rule for all partial effects of unemployment. This does not happen with surveys carried out on any other month.

As mentioned in Subsection 2.1, we also redid our analysis using only periods in which unemployment and the unemployment gap - measured as the difference between actual unemployment and the Congressional Budget Office's estimate of the non-accelerating-inflation rate of unemployment - moved in the same direction. Our findings are, if anything, strengthened, in that results by demographic groups show even starker differences between households in the upper and lower education and income groups. Finally, we split our sample between periods in which 12-month inflation was above $2 \%$, and periods in which it was below $2 \%$ and did a separate analysis for each subsample. Results are little changed.

\subsubsection{Probit estimates}

A natural alternative to our empirical approach would be to use probit models. In fact, the simple framework developed in Subsection 4.2 suggests precisely an probit-type specification. In that case, the signs of the partial effects, as well as statistical significance of the possible relationships between inflation and unemployment on one side and interest rates on the other side would be dictated by the Taylor rule coefficients on inflation and unemployment - which in turn determine the marginal effects in the probit. Thus, either all partial effects of inflation would come out in accordance with the principles underlying the Taylor rule, or they would all come out contradicting those principles. The same would be true of the partial effects of unemployment.

One advantage of an approach based on probits is that it imposes a parametric structure that uses information from all the empirical distributions of interest-rate responses given responses about inflation and unemployment to estimate the two coefficients that control those partial effects. In contrast, our nonparametric approach estimates each possible partial effect using only data from the two conditional distributions that define it, and this reduces the number of observations underlying the estimates. On the positive side, because our approach does not impose any structure on the data, it allows for partial effects that contradict each other. For example, we may estimate partial effects of inflation that are consistent with the stabilization principles underlying the Taylor rule when it comes to interest-rate increases, but which otherwise do not conform with those principles. In other words, the approach allows for asymmetries in perceptions about monetary policy that a standard probit would rule out by construction. Of course we could have modeled households' 
perceptions about the conduct of monetary policy by allowing explicitly for these asymmetries in the systematic component of households' interest-rate forecasts. However, that flexibility would have increased the number of parameters to be estimated one-to-one with the degrees of freedom that we wished to allow for in the estimation of partial effects. In the limit, if we wished to allow each partial effect to take any possible value, we would essentially be back to our nonparametric approach.

Table 15: Probits - Michigan Survey

\begin{tabular}{lccc}
\hline \hline & \multicolumn{2}{c}{ Dependent variables } \\
\multirow{2}{*}{ Inflation } & Interest rate up & & Interest rate down \\
\cline { 2 - 2 } & $0.119^{* * *}$ & & $-0.0918^{* * *}$ \\
& $(0.004)$ & $(0.005)$ \\
Unemployment & $0.021^{* * *}$ & \\
& $(0.006)$ & $(0.008)$ \\
\hline \hline
\end{tabular}

The table reports estimated coefficients and standard errors from probits of interest rates on inflation and unemployment. Coefficients followed by $* * *$ are significant at $1 \%, * *$ are significant at $5 \%$, and $*$ are significant at $10 \%$. Test statistics are based on Newey-West standard errors with correction for up to 12 lags.

Table 16: Probit Estimates - Michigan Survey, by demographics Dependent variable: Interest rate increases

\begin{tabular}{lcccc}
\hline \hline & \multicolumn{2}{c}{ Income } & \multicolumn{2}{c}{ Education } \\
Inflation & Low income & High income & No high school & College degree \\
& $0.132^{* * *}$ & $0.119^{* * *}$ & $0.140^{* * *}$ & $0.113^{* * *}$ \\
& $(0.010)$ & $(0.008)$ & $(0.014)$ & $(0.007)$ \\
Unemployment & $0.077^{* * *}$ & $-0.026^{* *}$ & $0.058^{* * *}$ & $-0.019^{*}$ \\
& $(0.015)$ & $(0.012)$ & $(0.020)$ & $(0.011)$ \\
\hline \hline
\end{tabular}

The table reports estimated coefficients and standard errors from probits of interest rates on inflation and unemployment. Coefficients followed by $* * *$ are significant at $1 \%,{ }^{*}$ are significant at $5 \%$, and * are significant at $10 \%$. Test statistics are based on Newey-West standard errors with correction for up to 12 lags.

To show that our main conclusions are robust to using probit models, in what follows we present results using this estimation method. Tables 15 and 17 present results for the Michigan Survey based on all pooled observations and for some demographic groups, respectively. They show that the relationship between inflation and interest rates implied by households' answers lines up well with our estimated partial effects of inflation. For unemployment, however, the estimated coefficient is not significant, which is consistent with the fact that we estimate conflicting partial effects of unemployment when we pool all household answers (Tables 4 and 5). When we split the sample by income and education levels, we find results that are consistent with the estimated partial effects reported in Table 6 . Note that the probit estimates can also speak to the asymmetry in 
Table 17: Probit Estimates - Michigan Survey, by demographics

Dependent variable: Interest rate decreases

\begin{tabular}{lcccc}
\hline \hline \multirow{4}{*}{ Inflation } & \multicolumn{2}{c}{ Income } & \multicolumn{2}{c}{ Education } \\
& Low income & High income & No high school & College degree \\
& $-0.092^{* * *}$ & $-0.099^{* * *}$ & $-0.094^{* * *}$ & $-0.104^{* * *}$ \\
Unemployment & $(0.012)$ & $(0.0094)$ & $(0.017)$ & $(0.008)$ \\
& $0.074^{* * *}$ & $0.170^{* * *}$ & $0.063^{* *}$ & $0.169^{* * *}$ \\
& $(0.020)$ & $(0.015)$ & $(0.026)$ & $(0.013)$ \\
\hline \hline
\end{tabular}

The table reports estimated coefficients and standard errors from probits of interest rates on inflation and unemployment. Coefficients followed by $* * *$ are significant at $1 \%, * *$ are significant at $5 \%$, and * are significant at $10 \%$. Test statistics are based on Newey-West standard errors with correction for up to 12 lags.

the responses regarding interest rate increases and decreases, which we detect with our estimated partial effects of unemployment. 